
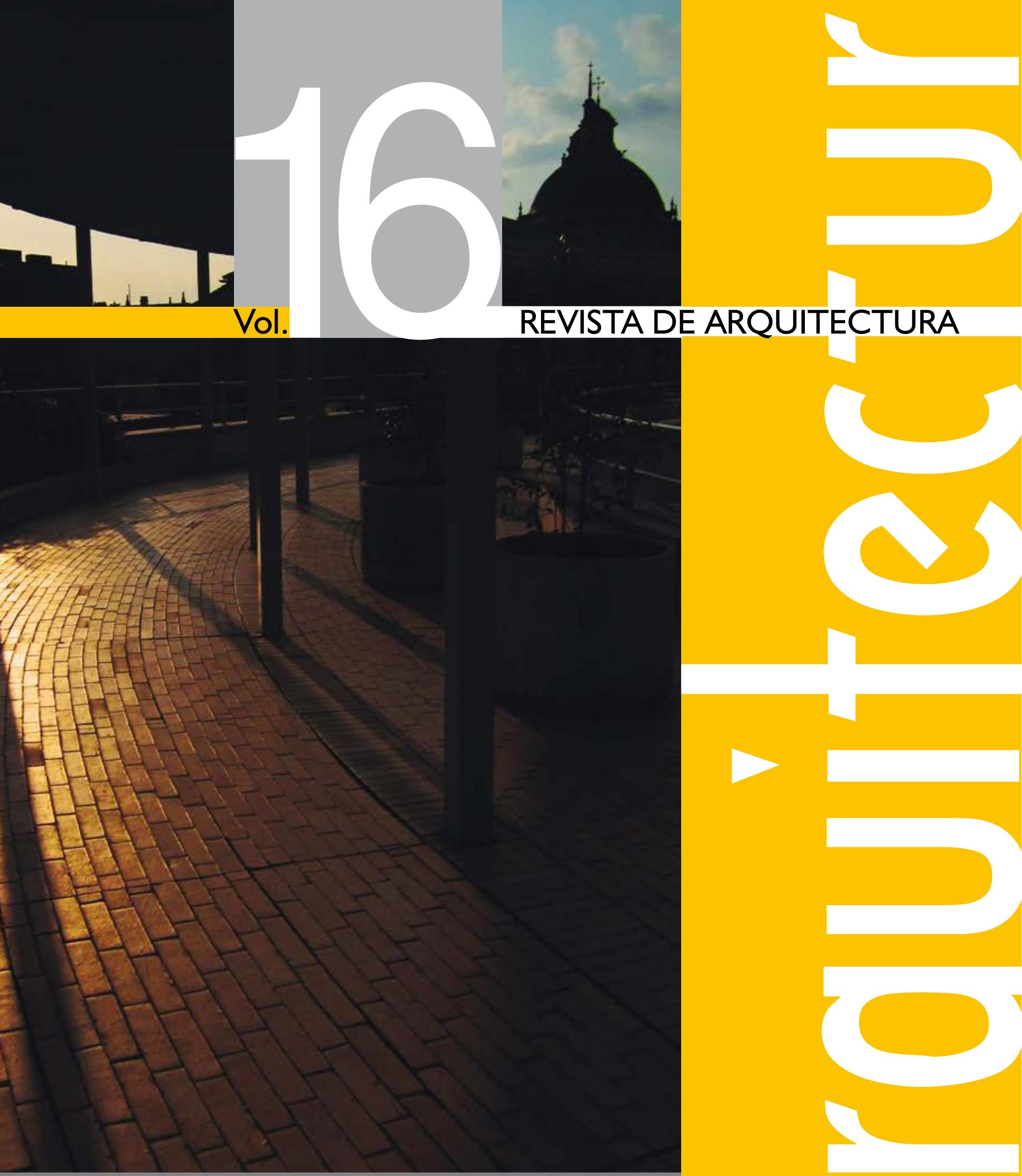
La Revista de Arquitectura (ISSN 16570308 impresa y ISSN 2357626X en línea) es una publicación seriada, arbitrada mediante revisión por pares, indexada y de libre acceso, donde se publican principalmente resultados de investigación originales e inéditos.

Está dirigida a la comunidad académica y profesional de las áreas afines a la disciplina (Arquitectura y Urbanismo). El primer número se publicó en 1999 y continúa con una periodicidad anual (enero-diciembre). Es editada por la Facultad de Diseño y el Centro de Investigaciones de la Facultad de Diseño -CIFAR- de la UNIVERSIDAD CATÓLICA DE COLOMBIA en Bogotá, Colombia.

La revista se estructura en tres secciones correspondientes a las líneas de investigación activas y aprobadas por la institución y una cuarta correspondiente a la dinámica propia de la Facultad de Diseño.

CULTURA Y ESPACIO URBANO. En esta sección se publican los artículos que se refieren a fenómenos sociales en relación con el espacio urbano, atendiendo aspectos de la historia, el patrimonio cultural y físico, y la estructura formal de las ciudades y el territorio.

PROYECTO ARQUITECTÓNICO Y URBANO. En esta sección se presentan artículos sobre el concepto de proyecto, entendido como elemento que define y orienta las condiciones proyectuales que devienen en los hechos arquitectónicos o urbanos, y la forma como estos se convierten en un proceso de investigación y nuevo de conocimiento. También se presentan proyectos que sean resultados de investigación, que se validan a través de la ejecución y transformación en obra construida de proceso investigativo. También se contempla la publicación de investigaciones relacionadas con la pedagogía y didáctica de la arquitectura, el urbanismo y el diseño.

TECNOLOGÍA, MEDIOAMBIENTE Y SOSTENIBILIDAD. En esta sección se presentan artículos acerca de sistemas estructurales, materiales y procesos constructivos, medioambiente y gestión, relacionados con el entorno social-cultural, ecológico y económico.
DESDE LA FACULTAD. En esta sección se publican artículos generados desde el interior de la Facultad de Diseño relacionados con las actividades de docencia, extensión o internacionalización, las cuales son reflejo de la dinámica y de las actividades realizadas por docentes, estudiantes y egresados; esta sección no puede superar el $20 \%$ del contenido con soporte investigativo.

Los objetivos de la Revista de Arquitectura son:

- Promover la investigación, el desarrollo y la difusión del conocimiento generado a nivel local, nacional e internacional.

- Conformar un espacio para la construcción de comunidades académicas y la discusión en torno a las secciones definidas.

- Fomentar la diversidad institucional y geográfica de los autores que participan en la publicación.

- Potenciar la discusión de experiencias e intercambios científicos entre investigadores y profesionales.

- Contribuir a la visión integral de la arquitectura, por medio de la concurrencia y articulación de las secciones mediante la publicación de artículos de calidad.

- Publicar artículos originales e inéditos que han pasado por revisión de pares, para asegurar que se cumplen con las normas de calidad, validez científica y ética editorial e investigativa.

- Fomentar la divulgación de las investigaciones y actividades que se desarrollan en la Facultad de Diseño de la UNIVERSIDAD CATÓLICA DE COLOMBIA.

Palabras clave de la Revista de Arquitectura: Arquitectura, diseño, educación arquitectónica, proyecto y construcción, urbanismo.

Key words for Revista de Arquitectura: Architecture, design, architectural education, urban planning, design-build.

Idiomas de publicación: Español, Inglés, Portugués

Título corto: RevArq
A El editor y los autores son responsables de los artículos aquí publicados.

Los autores son los responsables del material gráfico publicado.

Esta revista se acoge una licencia Creative Commons (CC) de Atribución - No comercial Compartir igual, 4.0 Internacional: "El material creado puede ser distribuido, copiado y exhibido por terceros si se muestra en los créditos. No se puede obtener ningún beneficio comercial y las obras derivadas tienen que estar bajo los mismos términos de licencia que el trabajo original".

Para más información:

http://co.creativecommons.org/tipos-de-licencias/

Universidad Católica de Colombia (2014, enero-diciembre). Revista de Arquitectura, 16. 1-144. ISSN: 1657-0308 E-ISSN 2357626X

(A)

Formato: $34 \times 24 \mathrm{~cm}$

Papel: Mate $115 \mathrm{~g}$

Tintas: Negro y policromía

Periodicidad: Anual
(A) CANje

OBJETIVO:

La Revista de Arquitectura está interesada en establecer canje con publicaciones académicas, profesionales o científicas, del área del Diseño, la Arquitectura y el Urbanismo o la educación superior, como medio de reconocimiento y discusión de la producción científica en el campo de acción de la publicación.

MECANISMO:

Para establecer Canje por favor descargar, diligenciar y enviar al correo electrónico de la Revista el formato:

RevArq FP20 Canjes

\section{A CONTACTO}

DIRECCIÓN POSTAL:

Avenida Caracas № 46 - 72. Universidad Católica de Colombia. Bogotá D.C.- Colombia

Código postal: 111311

Centro de Investigaciones (CIFAR).

Sede El Claustro. Bloque "L", 4 piso, Diag. 46a No. 15b - 10.

Arq. César Andrés Eligio Triana

Teléfonos: + 57 (1) 3277300 - 3277333

Ext. 3109; 3112 ó 5146

Fax: +57 (1) 2858895

CORREO ELECTRÓNICO:

revistadearquitectura@ucatolica.edu.co

cifar@ucatolica.edu.co

PÁGINA WEB:

www.ucatolica.edu.co Vínculo Publicaciones http://portalweb.ucatolica.edu.co/easyWeb2/arquitectura/pages.php/menu/319320363/id/2363/content/ revista-de-arquitectura/

EDITOR:

Mg. en Arq. César Andrés Eligio Triana celigio@ucatolica.edu.co

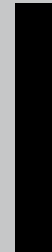


UNIVERSIDAD CATÓLICA DE COLOMBIA

PRESIDENTE

Édgar Gómez Betancourt

VICEPRESIDENTE - RECTOR

Francisco José Gómez Ortiz

VICERRECTOR JURÍDICO Y DEL MEDIO

UNIVERSITARIO

Edwin de Jesús Horta Vásquez

VICERRECTOR ADMINISTRATIVO

Édgar Gómez Ortiz

DECANO ACADÉMICO

Elvers Medellín Lozano

DIRECTORA DE INVESTIGACIONES

Elisa Urbina Sánchez

DIRECTORA EDITORIAL

Stella Valbuena García

\section{FACULTAD DE DISEÑO}

DECANO

Werner Gómez Benítez

DIRECTOR DE DOCENCIA

Jorge Gutiérrez Martínez

DIRECTOR DE EXTENSIÓN

Carlos Beltrán Peinado

DIRECTOR DE INVESTIGACIÓN

Juan Carlos Pérgolis

DIRECTOR DE GESTIÓN DE CALIDAD

Augusto Forero La Rotta

COMITÉ ASESOR EXTERNO

FACULTAD DE DISEÑO

Alberto Miani Uribe

Giovanni Ferroni Del Valle

Samuel Ricardo Vélez

Lorenzo Castro

\section{FACULTAD DE DISEÑO}

\section{Centro de INVESTIGACIONES - CIFAR}

\section{Andultecturo}

\section{REVISTA DE ARQUITECTURA}

DIRECTOR

Werner Gómez Benítez

EDITOR

César Andrés Eligio Triana

CONSEJO EDITORIAL

Werner Gómez Benítez

Jorge Gutiérrez Martínez

César Andrés Eligio Triana

Carlos Beltrán Peinado

Hernando Verdugo Reyes

EQUIPO EDITORIAL

COORDINADORA EDITORIAL

María Paula Godoy Casasbuenas mpgodoy@ucatolica.edu.co

DISEÑO Y MONTAJE

Juanita Isaza

juanaisaza@gmail.com

TRADUCTORA

Diana Carolina Peláez Rodríguez

dc.pelaez@uniandes.edu.co

CORRECTORA DE ESTILO

María José Díaz Granados M. mariajose_dgm@yahoo.com.co

PÁGINA WEB

Centro de investigaciones (CIFAR)

DISTRIBUCIÓN Y CANJES

Claudia Álvarez Duquino

calvarez@ucatolica.edu.co
COMITÉ EDITORIAL

(1)

TESPACIO URBANO

Sonia Berjman, PhD

ICOMOS, Buenos Aires, Argentina

Beatriz García Moreno, PhD

Universidad Nacional de Colombia. Bogotá, Colombia

Juan Carlos Pérgolis, MSc

Universidad Católica de Colombia. Bogotá, Colombia

René Julio Castillo, MSc PhD (Estudios)

Universidad del Rosario. Bogotá, Colombia

Proyecto aRQuitectónico y URbano

Hugo Mondragón López, PhD

Pontificia Universidad Católica de Chile. Santiago, Chile

Juan Pablo Duque Cañas, PhD

Universidad Nacional de Colombia. Bogotá, Colombia

Germán Darío Correal Pachón, MSc.

Universidad Católica de Colombia. Bogotá, Colombia

TECNOLOGÍA, MEDIOAMBIENTE Y SOSTENIBILIDAD

Luis Gabriel Gómez Azpeitia, PhD

Universidad de Colima. Colima, México

Luis Carlos Herrera Sosa, PhD

Universidad Autónoma de Ciudad Juárez, México

COMité CIENTífICO

Jorge Grané del Castillo, MSc Universidad de Costa Rica. San José, Costa Rica

Javier Peinado Pontón, MSc

Pontificia Universidad Javeriana. Bogotá, Colombia

Jorge Alberto Villamizar Hernández Universidad Santo Tomás. Bucaramanga, Colombia

Augusto Forero La Rotta, MSc

Universidad Católica de Colombia. Bogotá, Colombia

Luis Álvaro Flórez Millán, MSc

Universidad Católica de Colombia. Bogotá, Colombia

Elvia Isabel Casas Matiz, MSc

Universidad Católica de Colombia. Bogotá, Colombia 


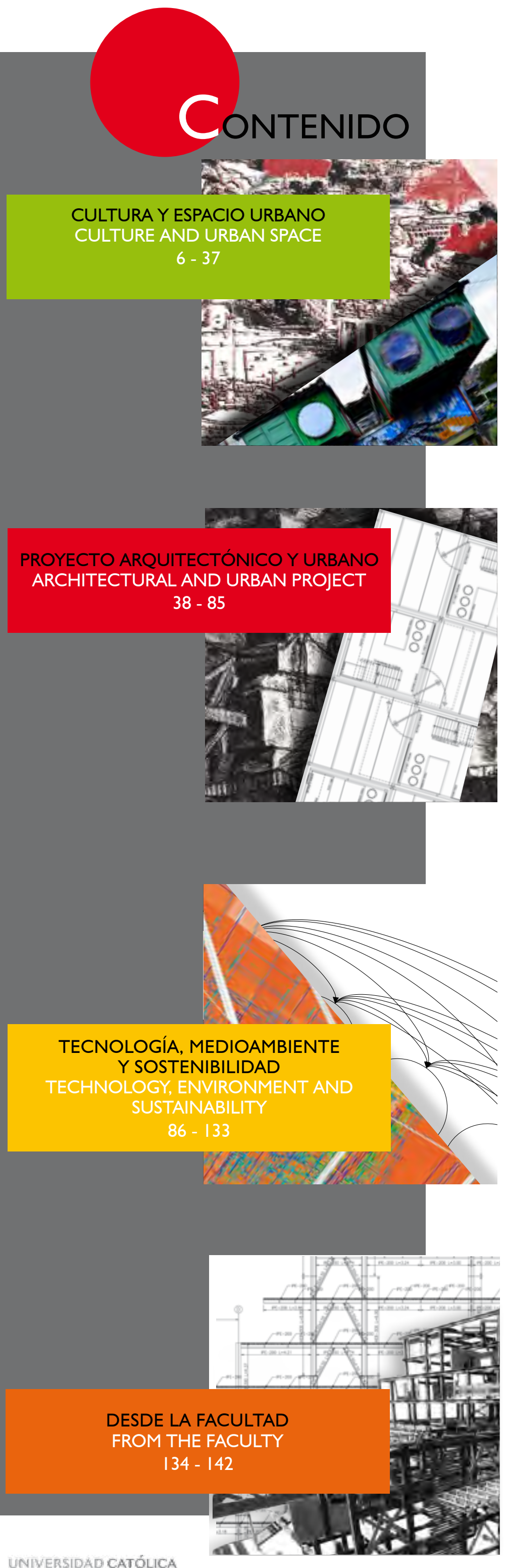

LOS USOS Y LA APROPIACIÓN DEL ESPACIO PÚBLICO PARA EL FORTALECIMIENTO DE LA DEMOCRACIA

PABLO PÁRAMO

ANDREA MILENA BURBANO

CIUDAD Y COMPROMISO CIUDADANO EN

LA HISTORIA DE OCCIDENTE

CARLOS ARTURO OSPINA HERNÁNDEZ

CARACTERIZACIÓN DEL MODELO DE APRENDIZAJE

A PARTIR DE LABORATORIOS DE DISEÑO CON

ÉNFASIS EN FACTORES SOCIALES

Álvaro JaVIER Bolaños Palacios

FABIÁN ADOLFO AgUILERA MARTÍNEZ

ANÁLISIS URBANO Y FORMAL DEL EDIFICIO

MIGUEL DE AGUINAGA

FELIPE VILLA MONTOYA

LEONARDO CORREA VELÁSQUEZ

VIVIENDAS DE EMERGENCIA EN URUGUAY

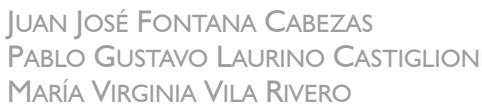

\section{CUESTIONES DE MÉTODO CREATIVO}

METAMORFOSIS Y CONCIENCIA MATERIAL EN LOS PROCESOS

CREATIVOS EN ARQUITECTURA

CARLOS IVÁN RUEDA PLATA

CRÍTICA SISTÉMICA

UN ENFOQUE HERMENÉUTICO DEL FENÓMENO

ARQUITECTÓNICO

ESKA ELENA SOLANO MENESES

TRADUCCIÓN DEL DISEÑO CONCURRENTE AL PROYECTO DE ARQUITECTURA

LUIS ÁLVARO FLóREZ MILLÁN

JaIRO HERnÁn OVAlLE Garay

LEONEL AUGUSTO FORERO LA ROTTA

PÁG. 77

EFICIENCIA DE ESTRATEGIAS DE ENFRIAMIENTO PASIVO EN CLIMA CÁLIDO SECO

LUIS CARLOS HERRERA SOSA

SIMULACIONES AMBIENTALES PARA LA SELECCIÓN DE MATERIALES EN DISEÑO DE ALOJAMIENTOS TEMPORALES EN CLIMAS TROPICALES

SARA LUCIANIM

A INFLUÊNCIA DAS PRATELEIRAS DE LUZ NO APROVEITAMENTO DA LUZ NATURAL SOB OBSTRUÇÃO EXTERNA

RICARDO NACARI MAIOLI

MARIANI DAN TAUFNER

CRISTINA ENGEL DE ALVAREZ

LA HABITABILIDAD COMO VARIABLE DE DISEÑO DE EDIFICACIONES ORIENTADAS A LA SOSTENIBILIDAD

ROLANDO ARTURO CUBILLOS GONZÁLEZ

JOHANNA TRUILLO

OSCAR ALFONSO CORTÉS CELY

Claudia Milena Rodríguez ÁlVAREZ

MAYERLY ROSA VILLAR LOZANO

LA SOSTENIBILIDAD DE LA VIVIENDA TRADICIONAL:

UNA REVISIÓN DEL ESTADO DE LA CUESTIÓN EN EL MUNDO

RIGOBERTO LÁRRAGA LARA

MIGUEL AGUILAR ROBLEDO

HUMBERTO REYES HERNÁNDEZ

JAVIER FORTANELLI MARTÍNEZ

$5^{\circ}$ CONCURSO DE DISEÑO EN ACERO PARA ESTUDIANTES DE ARQUITECTURA EN COLOMBIA - 2014

SEGUNDO PUESTO. MEMORIA

DiEGo AlEjANDRO MORA CASAS

JUAN CAMILO RINCÓN PULIDO

STEVEN GONZÁLEZ ZABALA

LUCAS PARDO MORA 


\title{
LA HABITABILIDAD COMO VARIABLE DE DISEÑO DE EDIFICACIONES ORIENTADAS A LA SOSTENIBILIDAD
}

\author{
Rolando Arturo Cubillos González, Johanna Trujillo, Oscar Alfonso Cortés Cely, \\ Claudia Milena Rodríguez Álvarez, Mayerly Rosa Villar Lozano \\ Universidad Católica de Colombia, Bogotá (Colombia) \\ Facultad de Diseño y Facultad de Ingeniería \\ Grupo de investigación "Sostenibilidad, Medio Ambiente y Tecnología (SOMET)"
}

\section{Cubillos González, R. A., Trujillo, J., Cor- tés Cely, O. A., Rodríguez Álva- rez, C. M. y Villar Lozano, M. R. (2014). La habitabilidad como variable de diseño de edificacio- nes orientadas a la sostenibili- dad. Revista de Arquitectura, 16, I14-125. doi: 10.14718/ RevArq.2014.16.13}

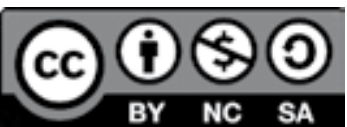

http://dx.doi.org/10.14718/ RevArq.2014.16.13

\section{Rolando Arturo Cubillos González}

Arquitecto, Universidad Nacional de Colombia.

Máster en Hábitat, Universidad Nacional de Colombia.

Líder grupo de investigación "Sostenibilidad, Medio Ambiente y Tecnología (SOMET)", Universidad Católica de Colombia.

racubillos@ucatolica.edu.co

\section{Johanna Trujillo}

Ingeniera Industrial, Universidad Católica de Colombia.

Máster en Ingeniería Industrial, Pontificia Universidad Javeriana.

Líder semillero Integración de la Cadena de Abastecimiento (InCas), Universidad Católica de Colombia.

jtrujillo@ucatolica.edu.co

\section{Oscar Alfonso Cortés Cely}

Arquitecto, Universidad La Gran Colombia.

Máster en Diseño bioclimático (candidato), Universidad ISTHMUS -COLIMA.

Docente investigador, Universidad Católica de Colombia.

oacortes@ucatolica.edu.co

\section{Claudia Milena Rodríguez Álvarez}

Ingeniera de sistemas, Universidad Nacional de Colombia.

Máster en sistemas y computación, Universidad de los Andes.

Docente investigador, Universidad Católica de Colombia. cmrodriguez@ucatolica.edu.co

Mayerly Rosa Villar Lozano

Arquitecta, Universidad Católica de Colombia

Magíster en Historia, Universidad Pedagógica y Tecnológica de Colombia

Docente investigador, Universidad Católica de Colombia.

mrvillar@ucatolica.edu.co

\section{RESUMEN}

Hoy, el crecimiento poblacional y el cambio climático afectan la óptima habitabilidad de las edificaciones. Por tanto, un mal diseño ocasiona que la habitabilidad no responda a los requerimientos de los usuarios y a las condiciones climáticas actuales. A partir del análisis del crecimiento poblacional y de los cambios climáticos que se están presentando en Bogotá, ies posible evaluar la calidad de la habitabilidad de las edificaciones $y$, por consiguiente, generar diseños óptimos que permitan que su uso sea sostenible en el tiempo y se adapten de manera adecuada a los cambios climáticos actuales? A fin de lograr esto, el análisis de la habitabilidad debe estar dirigido a diseñar edificaciones resilientes. Para ello, se propuso un Sistema de Gestión de la Información, que pretende generar modelos que estimen en el tiempo la adaptabilidad y la sostenibilidad de una edificación. La aproximación al concepto de habitabilidad plantea la interrelación de variables desde las visiones sociocultural y ambiental, entendida esta última como un sistema. Finalmente, se concluye que el prototipo de software en este momento se convierte en un laboratorio de exploración para la generación de otro software que pueda conducir a una patente de utilidad y aplicarlo en el ejercicio profesional.

PALABRAS CLAVE: sostenibilidad, flexibilidad, adaptabilidad, bioclimática, simulación, desarrollo de software.

\section{HABITABILITY AS DESIGN VARIABLE OF BUILDINGS HEADING TOWARDS} SUSTAINABILITY

\section{ABSTRACT}

Today, population growth and climate change affect the optimum habitability of buildings. Therefore, a bad design causes that habitability fails to respond the requirements of users and the current climate conditions. On the basis of the analysis of population growth and the climate changes taking place in Bogota, is it possible to evaluate the habitability quality of buildings and, consequently, create optimum designs that allow a sustainable use in time and adequate adaptation to current climate changes? In order to do so, the habitability analysis should be aimed at designing resilient buildings. To that end, the Information Management System created, intends to generate models that estimate a building's adaptability time and sustainability. The approximation of the habitability concept posits the interrelation of variables from the sociocultural and environmental views; the latter understood as a system. Finally, the article concludes that the software prototype at this time turns out to be an exploration laboratory for the generation of another software that could lead to a utility patent and be used professionally.

KEY WORDS: Sustainability, flexibility, adaptability, ecological design, simulation, software development.

\section{INTRODUCCIÓN}

El crecimiento poblacional y el cambio climático afectan la habitabilidad de las edificaciones. Por ello, estas enfrentan una alta demanda de recursos y energía que comprometen la sostenibilidad de las mismas. Por tanto, se genera un mayor impacto ambiental y se compromete la salud del hombre. Efectivamente, los edificios, al ser construidos, se convierten en una fuente indirecta de contaminación debido al consumo de recursos que requieren para su buen funcionamiento (Ramírez, 2002, p. 30).

Es por esto que el concepto de habitabilidad adquiere crucial importancia. Podemos definir la habitabilidad como la capacidad que tiene un edificio para asegurar condiciones mínimas de confort y salubridad a sus habitantes. En este orden de ideas, un mal diseño ocasiona que no se responda a las condiciones óptimas para que se mantenga la vida humana.

Entonces, las edificaciones requieren dentro de sus diseños elementos que recuperen el equilibrio con el ambiente para que puedan ser sostenibles. Asimismo, este equilibrio requiere de una gran capacidad de adaptabilidad a cambios extremos generados por el cambio climático, que le permitan garantizar las condiciones mínimas para la vida, es decir, garantizar una capacidad de resiliencia en las edificaciones. Por tanto, la respuesta a esta problemática debe estar dirigida a diseñar edificaciones resilientes, que permitan una adecuada solución a este fenómeno.

Esta investigación tiene como objetivo general: "proponer criterios para la evaluación de la calidad de la habitabilidad de las edificaciones en Bogotá".

Para dicho propósito, se formularon como objetivos específicos los siguientes:

- Proponer un modelo de comprensión del concepto de habitabilidad para orientar el diseño de edificaciones hacia la sostenibilidad.

- Diseñar una metodología para el diseño de indicadores de calidad habitacional desde las dimensiones social y ambiental.

- Diseñar una aplicación informática que permita la evaluación y el diseño de calidad habitacional en las edificaciones.

Como resultado de la investigación, este artículo científico expone los siguientes resultados: 
- En primer lugar, el modelo de habitabilidad orientado a la sostenibilidad (MHOS) (Cubillos-González, 2013), en donde se explica el concepto de habitabilidad como una variable de diseño de edificaciones orientadas a la sostenibilidad.

- En segundo lugar, se explica el método integral de diseño ambiental (MIDA) (Cortés y Villar, 2013), el cual establece rangos de confort según variables socioambientales para lograr una mejor habitabilidad en las edificaciones.

- Finalmente, se expone el proceso de desarrollo de la aplicación informática denominada Sistema de Gestión de Información de Proyectos de Vivienda Social (SGIPVIS), la cual permite la evaluación y el diseño de calidad habitacional en las edificaciones. Por último, se discute la evaluación y validación del módulo de simulación contenida en el software y su implementación a futuro.

\section{MetOdOLOGÍA}

El marco metodológico de esta investigación se construyó desde la perspectiva del pensamiento sostenible (Naciones Unidas, 1993). En primer lugar, se propuso la conceptualización del término habitabilidad a través de árboles de problemas para identificar las variables que determinan el concepto. Con esta herramienta, se organizó la información recolectada y se creó un modelo de relaciones causales que explican el proceso de habitabilidad en las edificaciones.

Luego, se utilizó la herramienta de identificación de patrones, con la cual se sistematizaron las variables analizadas en el árbol de problemas, obteniendo una descripción detallada de las características del factor de habitabilidad. Se encontró que una buena opción de análisis del factor de la habitabilidad en las edificaciones es la vivienda. Por tal razón, se valoró y validó un módulo de simulación del sistema de gestión de información de vivienda de interés social propuesto en la investigación "Diseño de prototipos flexibles de vivienda de interés social" (Cubillos-González, 2010).
Este módulo de simulación se propuso para que formara parte de otro módulo de simulación para el diseño de un prototipo de software. Sin embargo, se realizó una validación técnica que requiere de un ajuste para que el módulo del software sea operativo en un $100 \%$. Con la simulación se pudieron realizar diversos experimentos en un computador y validar la hipótesis a partir del estudio de diferentes escenarios. Para elegir una edificación las variables de decisión tuvieron los siguientes criterios:

a. El factor edificación presenta problemáticas para soportar las transformaciones realizadas por los usuarios a fin de lograr una óptima habitabilidad, ahí el usuario busca la flexibilidad o adaptabilidad como factor de decisión, para lo cual la edificación informal "se adapta en el tiempo", y la formal no cumple estos requerimientos (Camacol, 2009; Cubillos-González, 2006).

b. El costo, para lo cual la edificación formal dobla el costo de producción (DNP, 2009, p. 10).

c. La calidad, definida como la propone Escallón (2010a), debe ser diversa, flexible, suficiente, que construya ciudad y articulada.

d. La sostenibilidad de las edificaciones en el tiempo (Cubillos-González, 2010, p. 93).

e. Tamaño del lote (Cubillos-González, 2010, p. 93); individualización.

Según estos criterios, el hábitat se construye progresivamente así: áreas, habitaciones, espacios del edificio, manzanas y vecindarios; sin embargo, el alcance de esta investigación no tiene en cuenta la construcción de vecindarios, debido a que el control por parte del usuario de una vivienda llega tan solo a los espacios comunales y posiblemente hasta manzanas.

Por ejemplo, el proceso de habitabilidad de una vivienda formal o informal se da en las siguientes fases: compra, identidad, apropiación, necesidad, densificación o transformación, y renovación o reciclaje; en las primeras investigaciones del tema, el proceso se delimitó desde la identidad hasta la renovación del desarrollode unaedificación (Cubillos, 2006). En la figura 1 se explica detalladamente el proceso de habitabilidad actualizado.
จ Figura 1. Fases de una edificación y sus niveles de control

Fuente: adaptado por los autores de Cubillos (2006)

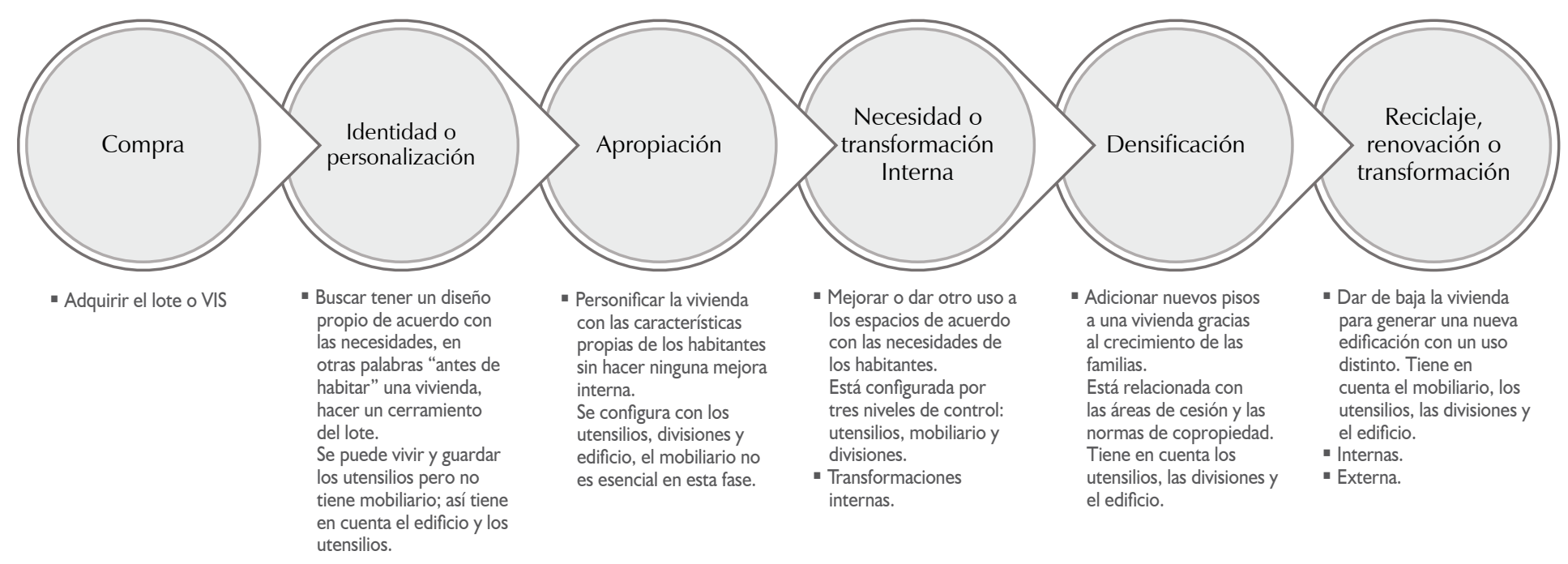




\begin{tabular}{|c|c|c|c|c|c|c|c|c|c|c|c|c|c|c|c|c|c|c|c|c|}
\hline \multirow{2}{*}{$\begin{array}{l}\text { Fase del diseño } \\
\text { Clases de hábitat } \\
\text { Áreas }\end{array}$} & \multicolumn{4}{|c|}{ Identidad } & \multicolumn{4}{|c|}{ Apropiación } & \multicolumn{4}{|c|}{ Necesidad } & \multicolumn{4}{|c|}{ Densificación } & \multicolumn{4}{|c|}{ Renovación } \\
\hline & U & & & & $U$ & & & & $U$ & M & & & $U$ & & & & $U$ & M & & \\
\hline Habitaciones & & & & & & & $\mathrm{D}$ & & & M & $D$ & & & & $\mathrm{D}$ & & & $M$ & $\mathrm{D}$ & \\
\hline Espacios comunales & & & & $\mathrm{E}$ & & & $\mathrm{D}$ & $\mathrm{E}$ & & & $\mathrm{D}$ & & & & $\mathrm{D}$ & $\mathrm{E}$ & & & $\mathrm{D}$ & $\mathrm{E}$ \\
\hline Manzanas & & & & $\mathrm{E}$ & & & & $E$ & & & & & & & & $\mathrm{E}$ & & & & $\mathrm{E}$ \\
\hline Vecindarios (N/A) & - & - & - & - & - & - & - & - & - & - & - & - & - & - & - & - & - & - & - & - \\
\hline
\end{tabular}

(A) Tabla 1. Interacción de

elementos del diseño

Fuente: los autores, con

información de Cubillos

(2006).

\begin{tabular}{|c|c|c|}
\hline Variable & Descripción & PARÁmetro \\
\hline Evento & Vivienda, hábitat o familia & $i=1,2 \ldots n$ \\
\hline $\begin{array}{l}\text { Inicio del proceso de } \\
\text { transformación }\end{array}$ & Momento inicial de la compra en tiempo cero & $0^{1}$ \\
\hline $\begin{array}{l}\text { Final del proceso de } \\
\text { transformación }\end{array}$ & $\begin{array}{l}\text { De acuerdo con el estudio de la universidad de los andes } \\
\text { y la universidad javeriana, el ciclo de vida es de } 12 \text { años, para } \\
\text { lo cual se identifica con una exponencial media } 12\end{array}$ & E(12) años \\
\hline $\begin{array}{l}\text { Tiempo promedio por etapa } \\
\text { de transformación }\end{array}$ & Final de proceso de transformación /7 etapas & \\
\hline $\begin{array}{l}\text { Etapa } 0,\left(E_{0}\right) \text { compra, área del } \\
\text { lote }(\text { al) }\end{array}$ & $\begin{array}{l}\text { Es un parámetro que indica el área del lote comprada, que es fijo } \\
\text { debido a que } 49 \text { es el área del lote ideal flexible en el cual podría } \\
\text { una persona vivir y llegar a adaptarse, para llegar a } 96 \mathrm{v} / \mathrm{h}\end{array}$ & 15,49 \\
\hline Etapa $1\left(E_{1}\right)$, identidad & Es el valor inicial con el que crece un lote & \\
\hline Etapa $2\left(E_{2}\right)$, apropiación & & 2 \\
\hline Etapa $3\left(E_{3}\right)$, necesidad & & $E_{3}=E_{1}+E_{2}$ \\
\hline Etapa $4\left(E_{4}\right)$, densificación & & $E_{4}=\frac{E_{1}+E_{3}}{2}$ \\
\hline Etapa $5\left(E_{5}\right)$, renovación & Es el área final máxima en la cual una vivienda podría llegar a crecer & $E(147) m^{2}$ \\
\hline \multicolumn{3}{|l|}{ Medidas de desempeño } \\
\hline Índice de construcción (ic) & & $I C=\frac{E 5}{A L}$ \\
\hline Área promedio (ap) & $\begin{array}{l}\text { Promedio área del lote y } 4 \text { etapas iniciales (identidad, } \\
\text { apropiación, necesidad, densificación) }\end{array}$ & $\frac{\sum_{i=1}^{5} E_{i}}{n}$ \\
\hline Área promedio por etapa (ape) & Promedio de las etapas 1 a la 5 & \\
\hline
\end{tabular}

Tabla 2. Variables de estudio

Fuente: los autores, con información de Cubillos (2006).
1 Iniciar una simulación en cero se da en simulaciones terminales, para el caso de vivienda no es terminal debido a que estocásticamente una vivienda no tiene un proceso secuencial, como se estima en esta investigación. 
Con lo anterior, las decisiones de diseño de una edificación comprenden el manejo de utensilios $(U)$, el mobiliario (M), las divisiones (D) y los edificios (E) (Cubillos-González, 2006), en la tabla 1 se explican detalladamente las interacciones y las fases del diseño.

Para la construcción del modelo de simulación de la investigación se utilizó la hoja de MS Excel, con el fin de estimar el crecimiento del área del lote de vivienda formal o informal; es importante aclarar que el modelo se basó únicamente en eventos donde las medidas de desempeño son extraídas del conjunto de sucesos del estado del arte.

Para el caso, cada corrida de simulación en el experimento equivale a viviendas y los eventos son las fases de diseño. Se utiliza la distribución t-student para los resultados. Las siguientes son las variables que se tuvieron en cuenta para el estudio (tabla 2).

Finalmente, se elaboró un prototipo de software utilizando la metodología ágil XP, la cual permitió el desarrollo de un software en un tiempo muy corto. Este trabajo se desarrolló con un equipo multidisciplinario de ingenieros de sistemas, arquitectos y estudiantes de la carrera de ingeniería de sistemas. Con la utilización del método ágil se enfatizó la comunicación entre los integrantes del equipo. Además, la investigación se desarrolló de manera racional y permitió identificar e incorporar las variables de habitabilidad en el software.

\section{RESULTADOS}

A continuación se realizará una síntesis de la explicación de los tres resultados obtenidos: el modelo de habitabilidad orientado a la sostenibilidad (MHOS), del método integral de diseño ambiental (MIDA) y, finalmente, se explicará el proceso de desarrollo del sistema de gestión de información de proyectos de vivienda social (SGIPVIS).

\section{PROPUESTA PARA EL DESARROLLO DE UN MODELO DE HABITABILIDAD ORIENTADO A LA SOSTENIBILIDAD (MHOS)}

La sostenibilidad es la relación entre el hombre y la naturaleza, en donde los patrones económicos y sociales deben estar en equilibrio para que no ejerzan presión al ambiente y no amenacen la existencia del hombre (Fiksel, Eason y Frederickson, 2012, p. 4). Las dimensiones que permiten dicho equilibrio son tres: la económica, la social y la ambiental. A su vez, las áreas de interrelación que se presentan en la sostenibilidad son tres: la eficiencia, la equidad y la habitabilidad.

La habitabilidad se puede definir como la capacidad que tiene un edificio para asegurar condiciones mínimas de confort y salubridad a sus habitantes. Esta surge de la relación entre la dimensión social con la dimensión ambiental en un hábitat construido. En este contexto, el uso eficiente de los recursos naturales se relaciona con la dimensión espacial para responder a las necesidades humanas. De esta interdependencia surge la habitabilidad como factor determinante en la construcción de una adecuada sostenibilidad.

En este orden de ideas, para entender el concepto de habitabilidad como un factor de sostenibilidad, se deben identificar los agentes que la afectan. En el tema de la habitabilidad se han identificado tres agentes:

- El crecimiento poblacional: el Departamento Nacional de Planeación (DNP) ha realizado estudios cuyos resultados han arrojado que el $80 \%$ de la población colombiana en las próximas décadas vivirá en ciudades (DNP, 2009) y este aumento poblacional se verá reflejado en un incremento de la demanda de edificaciones. La reducción espacial de las edificaciones y del espacio público, causado por la demanda del crecimiento poblacional, afecta la habitabilidad de la ciudad y no la hace sostenible. A su vez, la respuesta del mercado es una alta producción de vivienda en masa (Camacol, 2009), lo que ocasiona un alto consumo de recursos y energía para que la producción de edificaciones dentro de la ciudad sea viable.

- El cambio climático: hoy el planeta se enfrenta a un comportamiento del clima arbitrario, este tipo de conducta afecta directamente la habitabilidad de las edificaciones, porque se requiere que el diseño de las mismas tenga en cuenta este elemento para generar un tipo de edificación que las haga viables y habitables en el tiempo. En el caso de Bogotá, este fenómeno ha aumentado el riesgo en diferentes zonas (Alcaldía Mayor de Bogotá, 2012, pp. 3-4), sumado al impacto ambiental causado por la intervención del hombre. Esto, combinado con el proceso de cambio climático, representa un potencial riesgo de desastre (MAVDT, 2009, p. 14). Si no se controlan los actuales impactos ambientales, un gran número de edificaciones en diferentes áreas del país y de Bogotá se verán afectadas por fenómenos naturales, que serán acentuados por el cambio climático.

- El impacto ambiental: uno de los sectores de más alta contaminación es la construcción. Efectivamente, se ha calculado que la industria de la construcción consume entre un 47 a un $50 \%$ de los recursos mundiales (Edwards, 2001). Para materializar la 
idea del diseño de edificaciones sostenibles se requiere de una tecnología que las haga viables. Entonces, es necesario el estudio de diferentes tecnologías orientadas a la sostenibilidad, a fin de avanzar en las respuestas que reduzcan los efectos del impacto ambiental.

De acuerdo con los tres agentes anteriores, para que una edificación sea habitable debe satisfacer las siguientes necesidades de los usuarios:

- Necesidad de flexibilidad.

- Necesidad de que las edificaciones estén relacionadas con un mayor espacio público.

- Necesidad de accesibilidad a nivel arquitectónico y urbano.

- Necesidad de que las edificaciones estén relacionadas con una ciudad que gestione el consumo y el ahorro de energía.

- Necesidad de eficiencia en sus procesos de producción y gestión.

Es por esta razón que se debe incluir el concepto de resiliencia ampliamente debatido en la conferencia Río + 20 de las Naciones Unidas. La resiliencia se define como la capacidad que tiene un sistema para resistir diversas alteraciones sin que se afecte significativamente su estructura original. En el caso de las edificaciones, lo que se busca es que estas sean resilientes a los tres agentes que afectan la habitabilidad: el crecimiento demográfico, el cambio climático y el impacto ambiental.

Además, la mayoría de las edificaciones que se construyen en la ciudad no responde adecuadamente a la necesidad de habitabilidad de los usuarios. La mala utilización de materiales y la aplicación de métodos de construcción de alto impacto ambiental, causan el síndrome del edificio enfermo, definición propuesta por la Organización Mundial de la Salud (OMS).

En este sentido, el Building Research Establishment $^{2}$ (BRE) ha desarrollado una nueva herramienta para el cálculo del costo de la salud en la vivienda social de Gran Bretaña. La aplicación calcula los costos de salud y los peligros que se pueden presentar debido a malos diseños y al uso inadecuado de las viviendas.

Este ejemplo puede ser replicado en Colombia, ya que hoy se hace necesario evaluar y disenar herramientas que permitan la identificación de la variable de habitabilidad en las edificaciones para que puedan tener las condiciones adecuadas y respondan a la sostenibilidad. Esta evaluación podría realizarse, por ejemplo, a través del diseño de sistemas de gestión de información, los cuales permitirían cruzar datos y presentar un diagnóstico cercano a la realidad, el cual permita la toma decisiones para el diseño y la construcción de las edificaciones orientadas a la sostenibilidad.

En síntesis, el concepto de habitabilidad es primordial a la hora de diseñar edificaciones que estén orientadas a la sostenibilidad; para lograrlo, es importante que las edificaciones sean resilientes. Por tanto, se hace necesario evaluar la habitabilidad en las edificaciones para poder identificar un edificio resiliente que pueda responder al cambio climático por medio de principios de sostenibilidad.

Al respecto, en la actualidad se están desarrollando materiales con propiedades resilientes, como por ejemplo, pavimentos y concretos, por medio del cálculo del módulo de resiliencia, que permiten responder de manera óptima a los requerimientos de sostenibilidad (Quintana y Lizcano, 2007). Los impactos generados por los agentes que las afectan, y asimismo, determinar el grado de resiliencia que necesitan para ser sostenibles.

A partir de las ideas anteriores, se propuso un modelo teórico para la evaluación del factor de habitabilidad en las edificaciones sostenibles, denominado modelo de habitabilidad orientado a la sostenibilidad (MHOS). Este modelo combina las distintas variables descritas anteriormente, generando un sistema de comprensión del factor de habitabilidad, que permite evaluarlo identificando diferentes variables desde múltiples dimensiones.
$\Rightarrow$ Figura 2. Modelo de

habitabilidad orientado

a la sostenibilidad (MHOS)

Fuente: Cubillos-González y Rodríguez-Álvarez (2013).

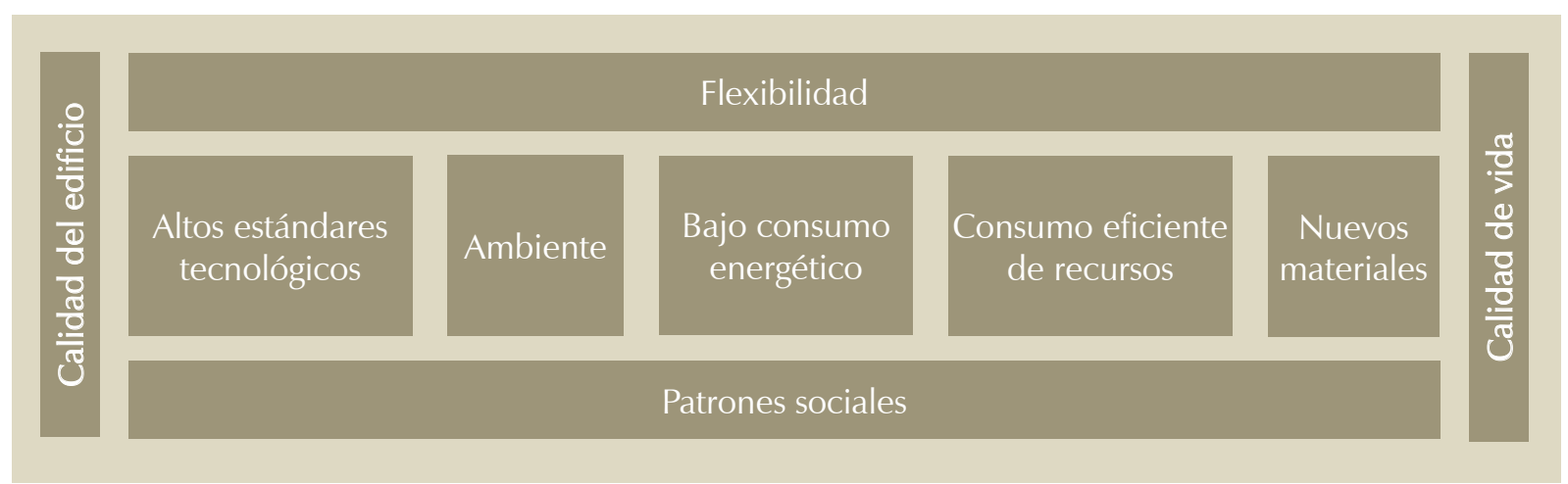

2 http://www.bre.co.uk/ 
La figura 2 muestra cuatro variables principales de color azul, que actúan como variables independientes: calidad del edificio, flexibilidad, calidad de vida y los patrones sociales. De estas cuatro variables se desprenden cinco variables dependientes: altos estándares tecnológicos, ambiente, bajo consumo energético, consumo eficiente de recursos y nuevos materiales.

\section{PROPUESTA DE UN MÉTODO INTEGRAL DE DISEÑO AMBIENTA (MIDA)}

El método integral de diseño ambiental (MIDA) está orientado de manera sistemática a establecer rangos de confort como resultado de la interacción de las variables socioambientales (figuras 2 y 3). Para lograr esto, este modelo vincula variables climáticas y ambientales en torno al concepto de habitabilidad y analiza el confort en la edificación.

Para determinar los rangos de confort se tienen en cuenta las variables climáticas que se interrelacionan con las condiciones más favorables de bienestar dentro de los procesos adaptativos que realizan los usuarios al interior de la vivienda. Por tanto, la variable ambiental cualifica el espacio interior cuando se concibe el diseño a partir de los parámetros climáticos del lugar y se establecen criterios como por ejemplo: la orientación, la asolación, la ventilación y la selección de materiales. También, se determinan las estrategias por implementar de acuerdo con la temperatura interior que debe tener la vivienda.

Asimismo, se evalúa cuál debe ser la humedad adecuada, la ventilación y los materiales que contribuyen a mitigar el calentamiento global. Es de vital importancia que cada parámetro climático y social se evalúe con datos estadísticos y confrontarlos con la realidad a través de trabajo de campo, así como determinar las tipologías habitacionales desde su déficit cualitativo. En la figura 3 se presenta gráficamente la interrelación de variables.

Las variables sociales analizan el componente habitacional y sus principales servicios públicos, que son los que garantizan las condiciones mínimas de salubridad y confort. En cuanto a las variables climáticas, se analizan las estrategias de climatización pasiva, a fin de lograr el objetivo de encontrar la sinergia entre los elementos sociales y ambientales.

En términos de eficiencia se analizaron las mínimas condiciones de adaptabilidad y ahorro energético que se generan a partir de los materiales y el uso de sistemas pasivos de climatización, en conjunto con las condiciones ambientales del lugar de implantación (Serra, 2010, p. 13).

Por tanto, los procesos de adaptabilidad se logran desde la flexibilidad del diseño en concordancia con los parámetros socioambientales y su acondicionamiento al contexto físico, social y cultural. En síntesis, a partir de la aplicación de estas estrategias, el diseño de una edificación orientada a la sostenibilidad apunta a generar un hábitat más humano y flexible en términos de diseño y eficiencia energética.

\section{DESARROLLO DEL SISTEMA DE GESTIÓN DE INFORMACIÓN DE PROYECTOS DE VIVIENDA SOCIAL (SGIPVIS)}

A partir de los modelos anteriores, y después de realizar el análisis de los requerimientos del sistema SGIPVIS, se procedió a realizar el diseño

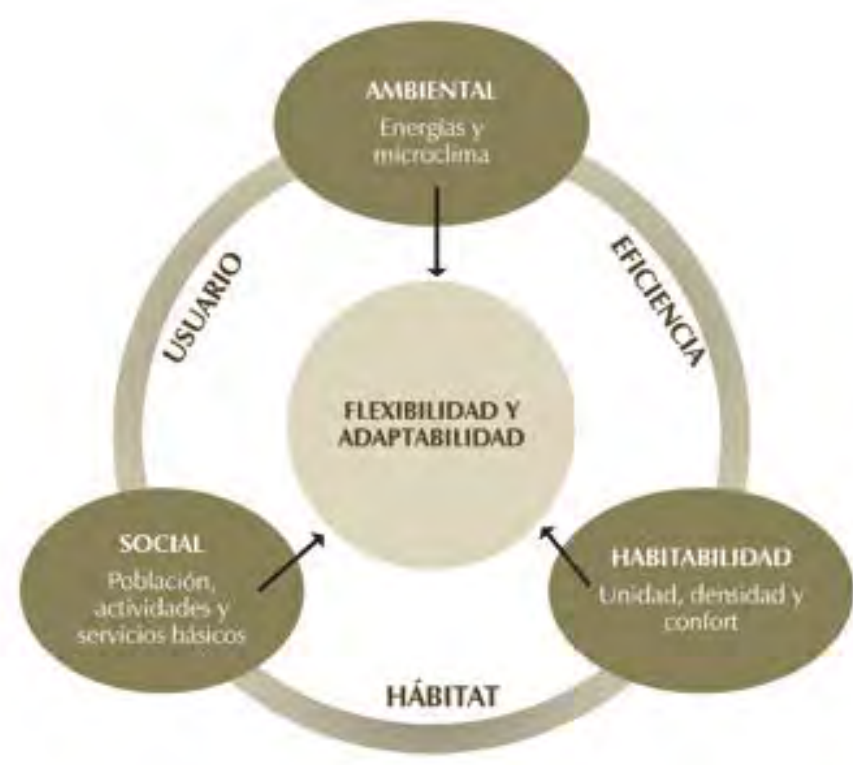

integral de diseño ambiental (MIDA)

Fuente: Cortés y Villar (2013).

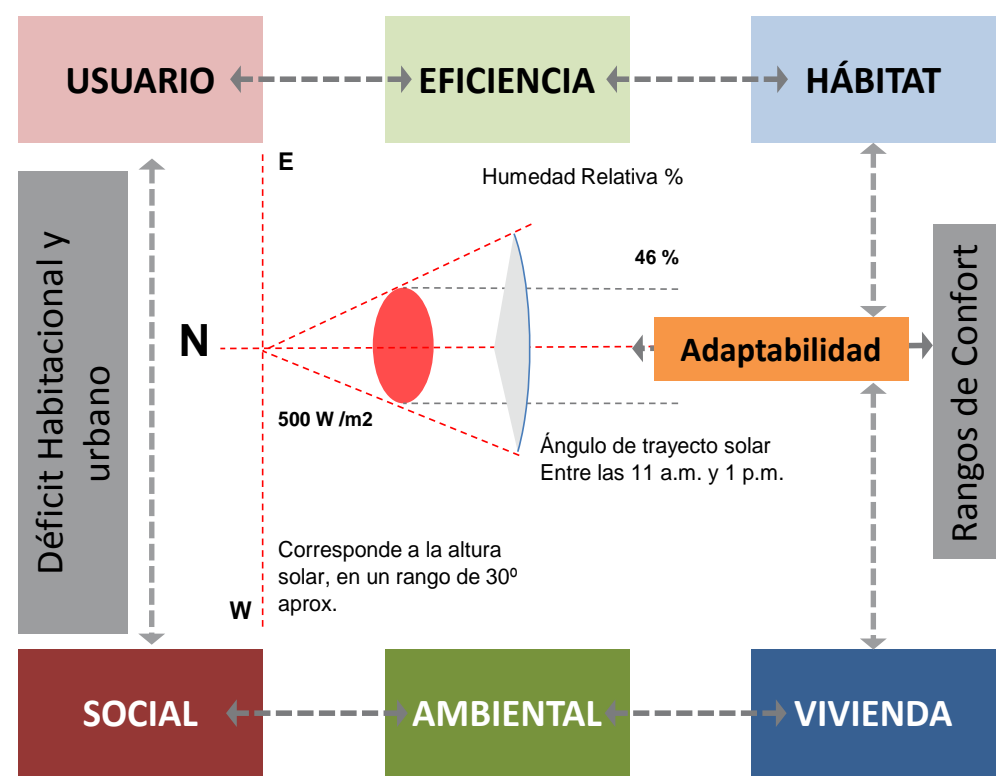

A Figura 4. Diagrama de interrelación de variables

Fuente: Cortés y Villar (2013). 


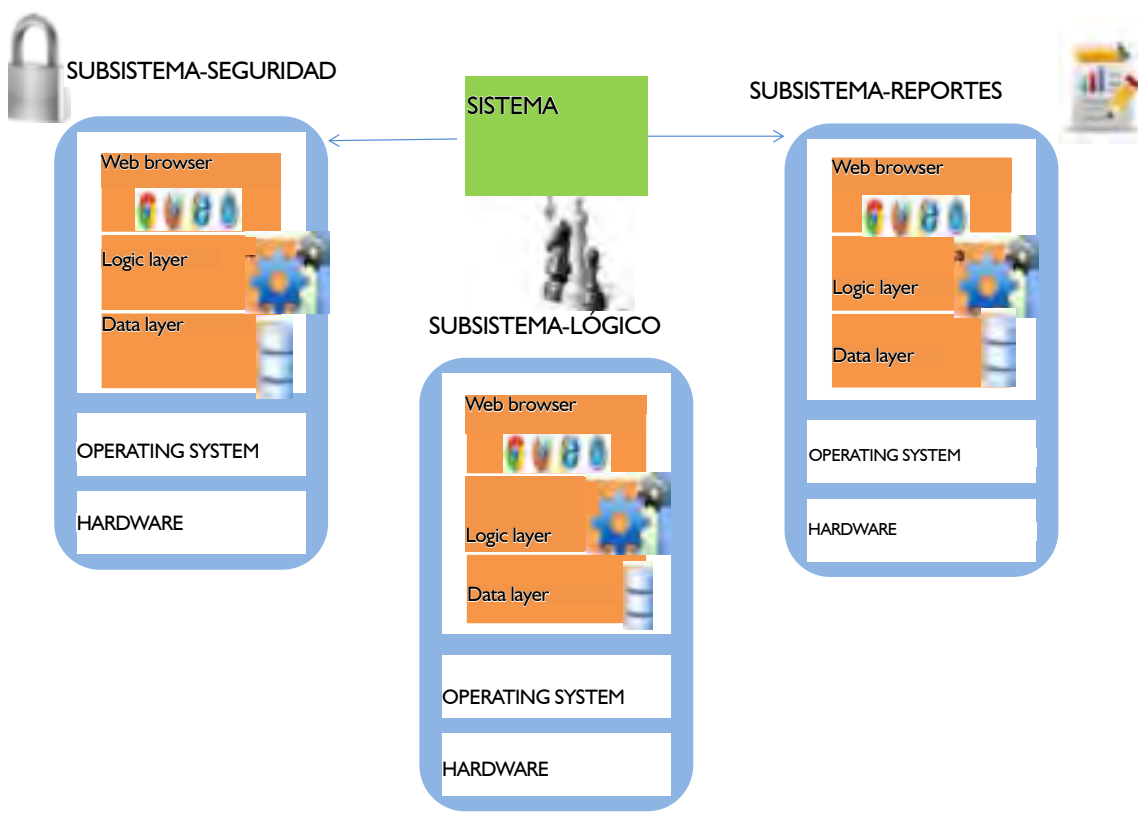

A Figura 5. Arquitectura del sistema SGIPVIS Fuente: Pestana (2013).

del sistema de gestión de información. La arquitectura del sistema se dividió en tres subsistemas (figura 5). El primero tenía como objetivo controlar la seguridad del sistema en el acceso y la restricción de sus contenidos. El segundo tenía como objetivo guardar los requerimientos funcionales del sistema de gestión de información. Por último, el subsistema de reportes tenía como objetivo exportar en diferentes formatos la información relevante y necesaria para los usuarios del prototipo de software.

\section{IMPLEMENTACIÓN Y PRUEBA DE RESULTADOS DEL SISTEMA DE GESTIÓN DE INFORMACIÓN}

La implementación del SGIPVIS tuvo dos fases. En la primera, se desarrolló un sistema para el diseño de los requerimientos de usuario, con la implementación de una página web. La segunda fase desarrolló el sistema SGIPVIS, donde se incluyeron los tres subsistemas mencionados. Para la implementación de los dos sistemas se utilizó el lenguaje Groovy con el framework Grails para la implementación web.

Uno de los elementos más importantes dentro del SGIPVIS es la funcionalidad de simulación de proyectos arquitectónicos en el área de la vivienda. Para ello se realizaron pruebas a fin de comprobar la funcionalidad del módulo de simulación.

Para comprobar la funcionalidad del simulador de flexibilidad, se realizó la prueba para un proyecto de vivienda social de 20 viviendas, evaluado con un tiempo mínimo de simulación de 10 años y un tiempo máximo de 25 años, para un área inicial de 60 metros cuadrados.

Una vez realizada la prueba, se observó que los resultados de los datos podrían acercarse a la realidad en cuanto al crecimiento que tiene la vivienda social en Bogotá con respecto a su área inicial, comprobando la funcionalidad del simulador. Sin embargo, fue necesario evaluar por aparte y más a fondo el diseño del proceso de simulación estocástica trazada para el módulo, razón por la cual se realizó una evaluación del módulo de simulación. Esta se llevó a cabo en una hoja de MS Excel en donde se implementó el diseño de simulación estocástica.

\section{Evaluación y validación del módulo de simula- ción del sistema de gestión de información}

Para la validación del módulo de simulación se realizaron cuatro experimentos, para un horizonte de planeación de 10 hectáreas; los parámetros de cada etapa se muestran en la tabla 3:

El modelo pretendía ser estocástico, como se explica a continuación (figura 6), donde una etapa previa en un estado i, tiene una probabilidad asociada al estado del arte de la normatividad para pasar a la etapa siguiente, i+1 (Liu et al., 2013; Rigaux, Carlin, Nguyen-thé y Albert, 2013; Solibakke, 2012). A partir de esto, se identificó en la validación que el modelo en MS Excel se encontraba mal parametrizado, por lo cual se recomendó generar un modelo de simulación de datos recolectados en campo que represente la realidad de la toma de decisiones de los usuarios de las edificaciones y se use la simulación para representar la realidad característica principal de esta herramienta.

\section{DisCUSIÓN}

\section{RECOMENDACIONES PARA EL MODELO DE SIMULACIÓN PROPUESTO}

A partir de la evaluación realizada al módulo de simulación se identificó que, en su elaboración, se desconocieron los estados como procesos estocásticos, llamados también sucesiones de eventos, definidos como el resultado de un conjunto de procesos que en el tiempo presentan etapas dependientes del azar o que tienen asociadas una probabilidad de ocurrencia.

El caso más simple de los procesos estocásticos son las Cadenas de Markov, que son una sucesión de ensayos u observaciones en la cual cada ensayo tiene el mismo número finito de resultados del resultado del ensayo inmediatamente precedente y no de cualquier resultado previo (Janczura y Weron, 2012; Li, Singh y Mishra, 2013).

Por consiguiente, para el ajuste de la matriz de transición se recomienda una etapa de validación del modelo. Es así como se debe registrar la probabilidad de pasar de un estado a otro, en donde es pertinente implementar en el modelo de simulación las columnas que indican el estado 


\begin{tabular}{|c|c|c|c|c|c|c|c|c|c|}
\hline \multirow{2}{*}{ Experimento de simulación } & \multirow{2}{*}{ Densidad } & \multicolumn{6}{|c|}{ Tamaño del lote por etapa ()$^{3}$} & \multirow[t]{2}{*}{ Normativa } & \multirow[t]{2}{*}{ Tipo } \\
\hline & & 0 & 1 & 2 & 3 & 4 & 5 & & \\
\hline 0 & $96 \mathrm{v} / \mathrm{h}$ & & & & & & & & Informal \\
\hline $\begin{array}{l}\text { 1. Ciudadela El Recreo, } \\
\text { Bogotá - Metro vivienda }\end{array}$ & $240 \mathrm{v} / \mathrm{h}$ & 25 & 37,5 & 50 & 62,5 & 75 & 75 & & Formal \\
\hline $\begin{array}{l}\text { 2. VIS }{ }^{4} \text { en agrupación } \\
\text { según POT }\end{array}$ & $200 \mathrm{v} / \mathrm{h}$ & 15 & 15 & 24,64 & 40,71 & 50,35 & 60 & $\begin{array}{l}\text { Decreto } 619 \text { de } \\
\quad 2000\end{array}$ & Formal \\
\hline 3. VIS en lote individual & $108 \mathrm{v} / \mathrm{h}$ & 35 & 28 & 47,25 & 66,5 & 85,75 & 105 & $\begin{array}{l}\text { Decreto } 2060 \text { de } \\
2004\end{array}$ & Formal \\
\hline 4. Lote individual flexible & $96 \mathrm{v} / \mathrm{h}$ & 49 & 36,75 & 73,5 & 110,25 & 128,63 & 147 & Propuesta & Formal \\
\hline
\end{tabular}

3 Se intenta simular los procesos de las etapas con una distribución uniforme entre parámetros (etapa 0 tamaño del lote en metros, etapa 5 tamaño flexible o normativo en metros), pero la metodología no es replicable debido a que nunca se indica qué tipo de distribución sería, y de dónde se toman los parámetros. Lo correcto sería aplicar una distribución empírica y no uniforme basada en Cadenas de Markov. No se entienden los cálculos en la hoja de simulación de Excel, no se describe la forma de cálculo de esas distribuciones en el conjunto de documentos revisados de la investigación. Por tanto, en la lectura de los documentos de investigación se observó que en algunos momentos del proceso se intentó describir el cálculo a partir de la segunda fila de distribuciones uniformes del modelo.

A Tabla 3. Horizonte de planeación de 10 hectáreas

Fuente: los autores, con información de Cubillos (2012b).

4 Vivienda de Interés social.

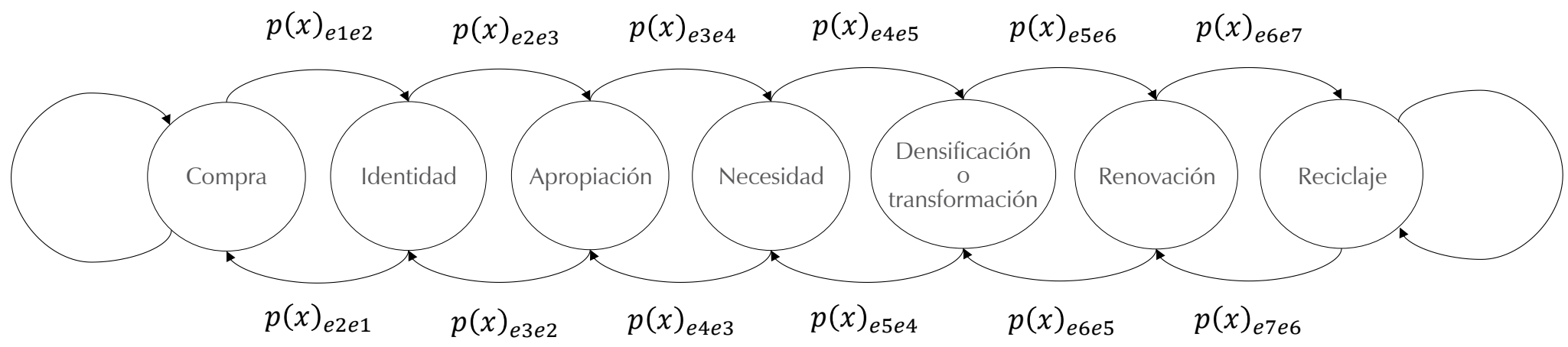

A Figura 6.

Representaciones de los nodos de transición para el proceso estocástico de vivienda

Fuente: los autores con base en Kahrobaee y Asgarpoor (2013), Lai
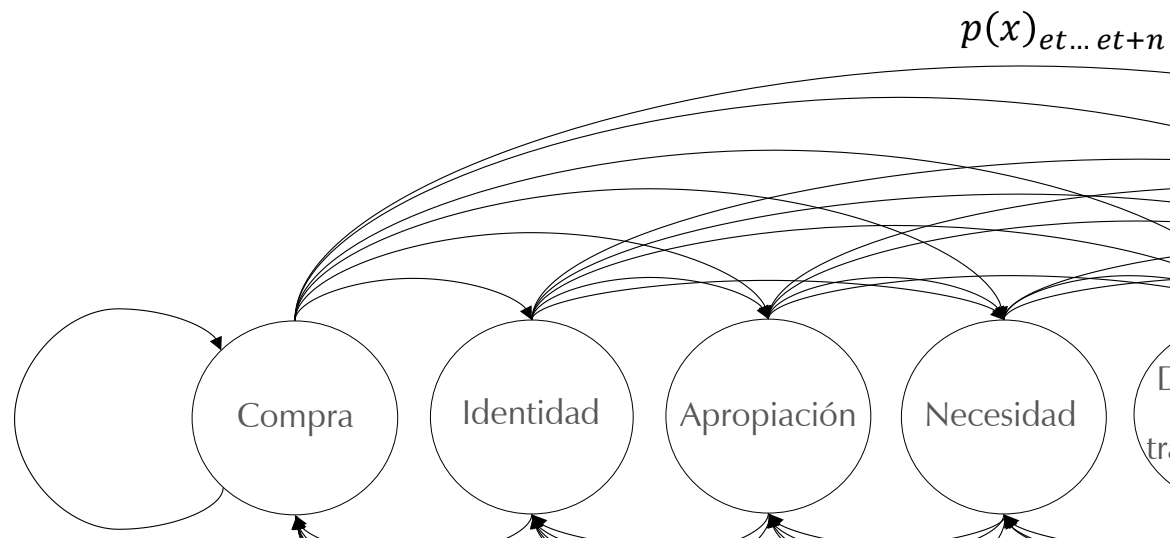
Chung (1983).

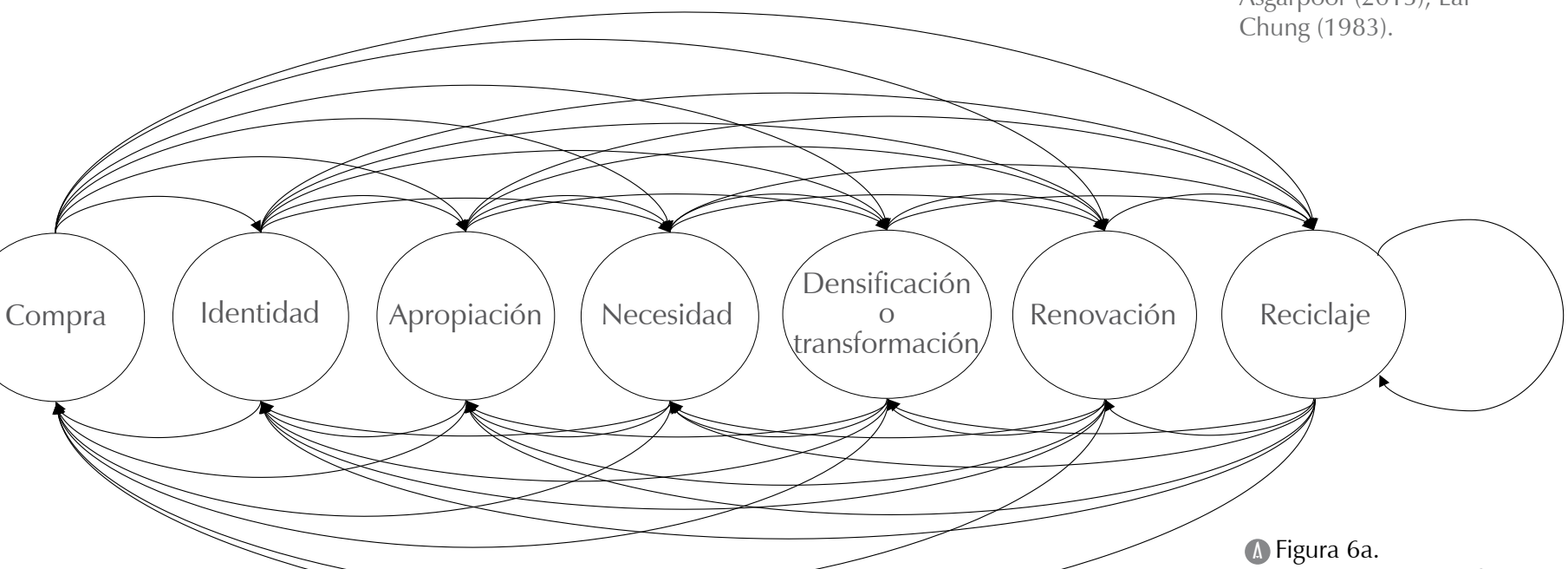

Representaciones de nodos de transición para el $p(x)_{e t-1 \ldots e t}$ proceso estocástico de vivienda

Fuente: los autores. 
Tabla 4. Matriz de transición para procesos estocásticos de vivienda

Fuente: los autores.

\begin{tabular}{|c|c|c|c|c|c|c|c|}
\hline $\begin{array}{c}\text { Estado } \\
\text { inicial-final }\end{array}$ & Compra & Identidad & Apropiación & Necesidad & $\begin{array}{l}\text { Densificación } \\
\text { o } \\
\text { transformación }\end{array}$ & Renovación & $\begin{array}{l}\text { Reci- } \\
\text { claje }\end{array}$ \\
\hline Compra & NA & 0,3 & 0,05 & 0,3 & 0,2 & 0,1 & 0,05 \\
\hline Identidad & 0,8 & NA & & & & & \\
\hline Apropiación & & & NA & & & & \\
\hline Necesidad & 0,7 & & & $\mathrm{NA}$ & & & \\
\hline $\begin{array}{l}\text { Densificación o } \\
\text { transformación }\end{array}$ & & & & & NA & & \\
\hline Renovación & & & & & & NA & \\
\hline Reciclaje & & & & & & & NA \\
\hline
\end{tabular}

Procedimiento para la simulación

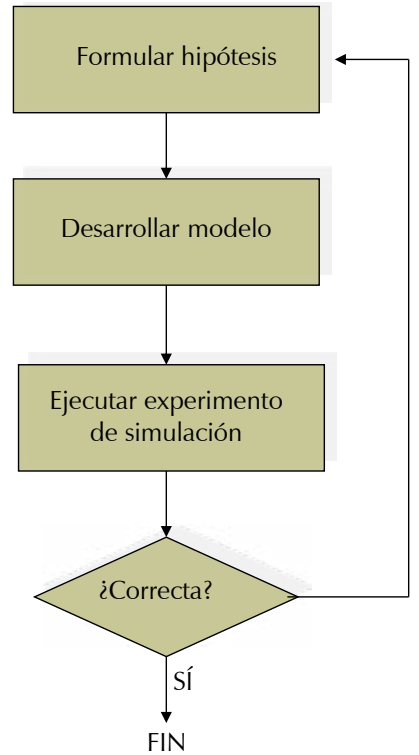

A $>$ Figura 7 .

Recomendaciones para el modelo de simulación propuesto Fuente: los autores.
Modelo de simulación discreto

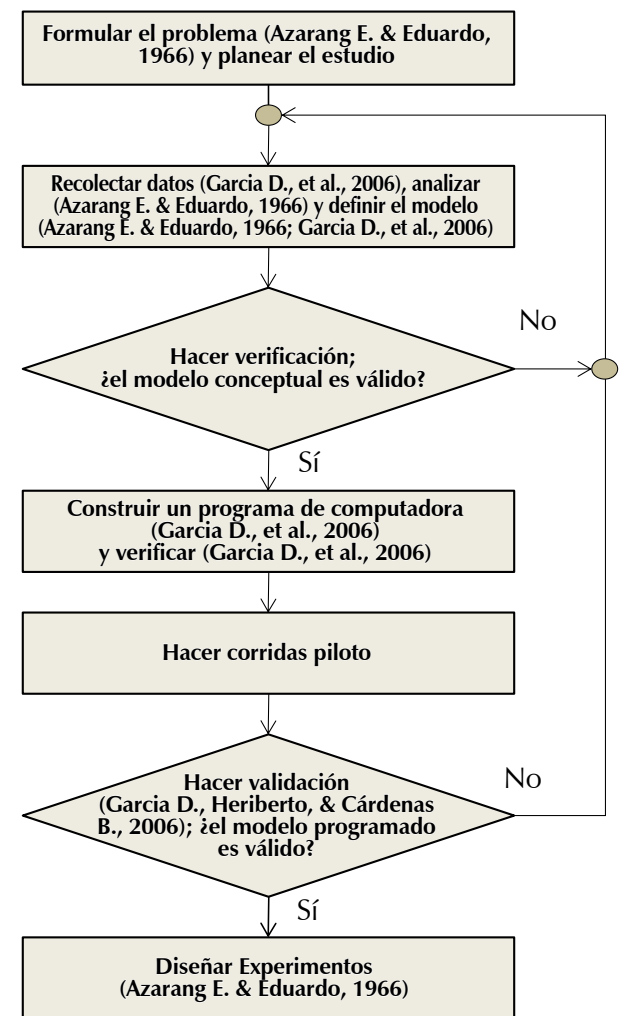

Realizar trabajos de producción

Analizar datos de salida

inicial y las filas del estado final (Cameron y Pettitt, 2012; Dotto et al., 2011). Por tanto, la matriz propuesta debe estar balanceada de forma horizontal (por filas), y su sumatoria debe ser el $100 \%$; por columnas no hay restricción.

La tabla 4 muestra que la probabilidad de que una familia pase de un estado de compra a un estado de necesidad es del $70 \%$, y del estado de necesidad a compra es del $30 \%$; y así sucesivamente. Se recomienda ser rigurosos porque en el proceso de investigación no se muestra la

construcción de estos estados, se hace de manera lineal estimando secuencialmente el modelo desde compra hasta reciclaje.

Si se quiere ver el esquema en forma de red, entonces se construye como se muestra en la figura 6, para facilitar a los investigadores en arquitectura su entendimiento; cada nodo es un estado y cada flecha indica la probabilidad de cambio de estado. La Cadena de Markov hace que el modelo exprese la realidad y el conjunto de todas las interacciones o decisiones que un usuario pueda tener en un instante de tiempo; así, una persona en un estado i, podría pasar a cualquier otro estado i+n (Kahrobaee y Asgarpoor, 2013; Mateescu y Serwe, 2013; Solibakke, 2012).

\section{RECOMENDACIONES PARA LA RECOLECCIÓN DE DATOS}

El planteamiento del proceso de simulación propuesto por Flores (2010), no sigue la metodología de un estudio de Simulación Discreto clásico (Sang, et al., 2011); por tanto, la fase de recolección de datos en el sector real y su validación con este sector como herramienta para estimación de las medidas de desempeño del proceso de las edificaciones se debe hacer como se muestra en la parte derecha de la figura 7 .

Para esta investigación aún están por desarrollar muchos elementos, su sustento teórico son los decretos 619 de 2000 y 2060 de 2004, para lo cual las entidades interesadas en implementar el modelo podrían ser la Secretaría de Hábitat, y el mercado objetivo del software son las constructoras de vivienda de interés social (VIS).

El modelo puede ser de mucha utilidad para diferentes agentes interesados en el tema. Por ejemplo, un usuario podría elegir la constructora con la que pretende comprar su vivienda de acuerdo con sus necesidades de flexibilidad. Sin embargo, la investigación puede llegar a la aplicación y formulación de políticas públicas desde la perspectiva de gestión urbana. 


\section{MEDIDAS DE DESEMPEÑO DEL MODELO}

Con el fin de generar medidas de desempeño reales (figuras 8 y 9), se debe hacer una recolección de datos en campo con un muestreo por estratos socioeconómicos debido a que el modelo está basado en la flexibilidad de la vivienda formal e informal, eso indica que no es únicamente para un estrato socioeconómico determinado.

Luego de estratificar la muestra por tipo de vivienda, se aplica un muestreo aleatorio simple
(MAS) por cada nivel de estudio, con el fin de cumplir los supuestos de extender los resultados a la población y los de aleatoriedad, independencia y uniformidad básicos de un modelo de simulación clásico. La identificación de parámetros se debería hacer con la metodología propuesta en la figura 8 .

Luego de identificar los parámetros se procede nuevamente a formular la simulación en una estructura multivariante (Yang et al., 2011) si se va a formular en MS Excel.
จ Figura 8. Etapas de recolección de datos y variables para el muestreo estratificado y aleatorio simple

Fuente: los autores.
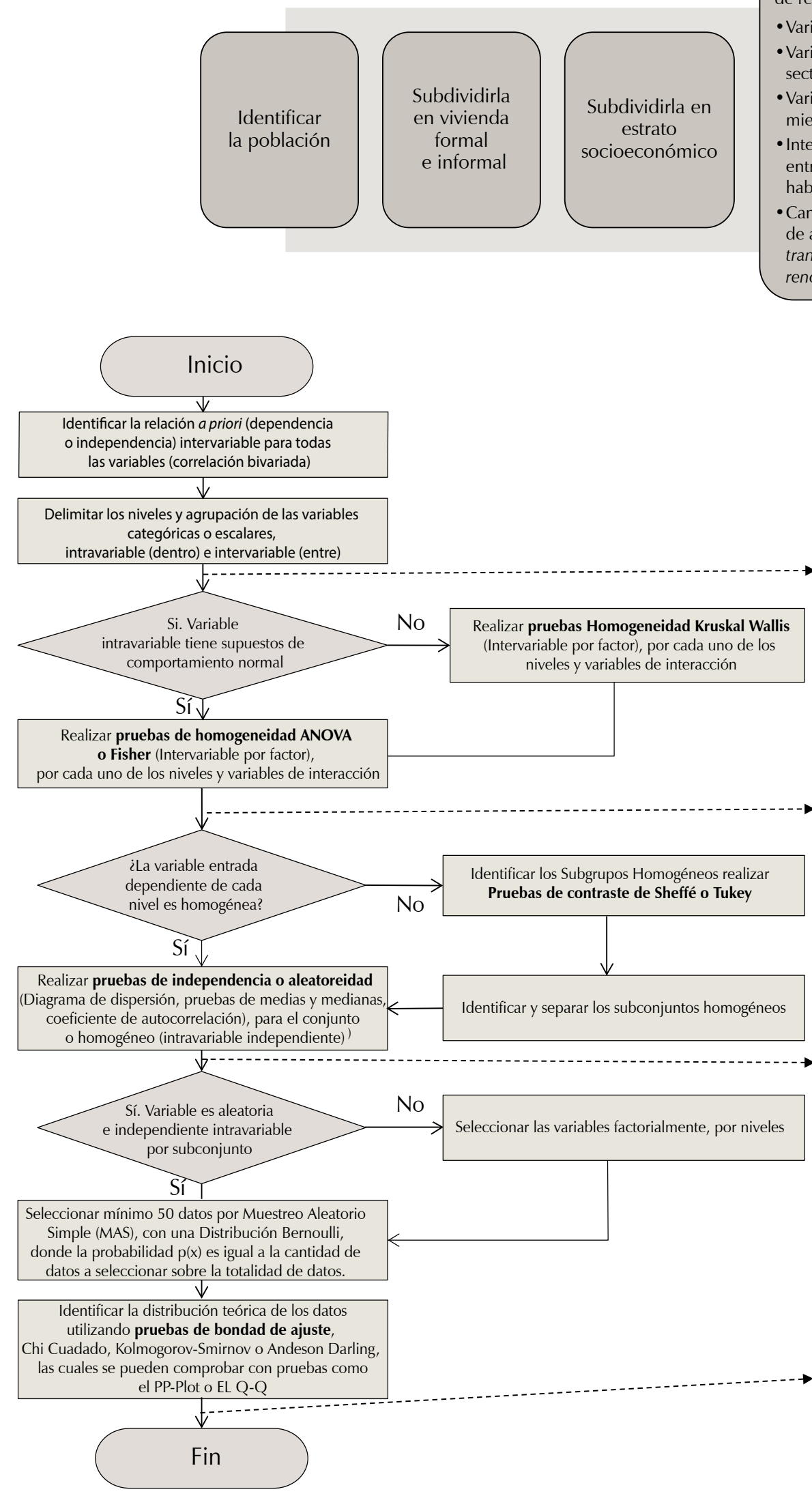

Diseñar el instrumento de recolección

- Variable costo - Variable ubicación o sector

- Variable cantidad de miembros por familia - Intervalo de tiempo entre etapas habitables

- Cantidad, costo y tipo de adaptaciones, transformación, renovación y reciclaje

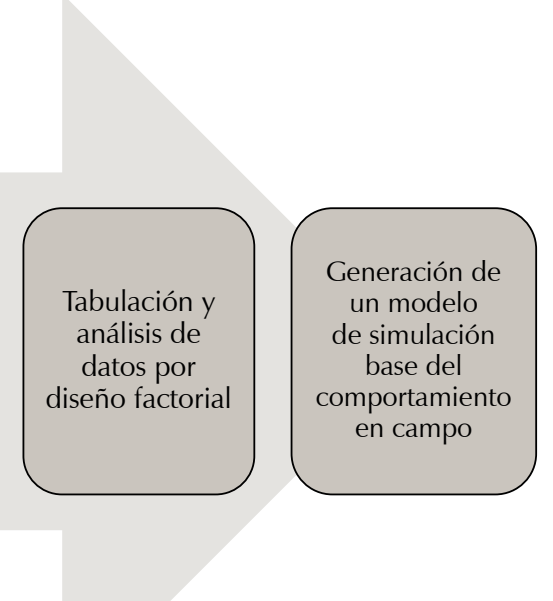

Figura 9. Metodología de análisis de datos Fuente: Trujillo y Cubillos (2014); Trujillo, González y Velásquez (2013); Trujillo, Vallejo y Becerra (2010).
Generar una matriz nxn, donde $\mathrm{n}$ son todas las de variables y cada cruce de celda indica la relación intervariable (entre variables)
Generar una matriz nxn, donde $\mathrm{n}$ son todas las de variables intra e interdependientes y cada cruce de celda indica la "homogeneidad por cada variable"
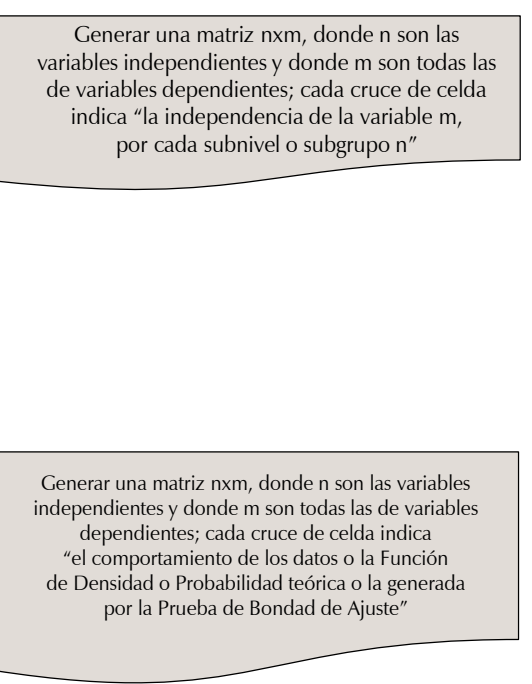

Generar una matriz nxm, donde $\mathrm{n}$ son las variables dependientes $y$ donde $m$ son todas las de variables comportamiento de los datos o la Función pridad o Probabilidad teórica o la gener de Bondad de Ajuste" 
La siguiente es la fórmula del muestreo estratificado (Sheaffer, Mendenhal y Ott, 2007):

$$
n=\frac{\sum \frac{N_{i} P_{i} Q_{i}}{W_{i}}}{N^{2} \frac{e^{2}}{4}+\sum N_{i} P_{i} Q_{i}}
$$

Donde,

$$
W_{i}=\frac{N_{i}}{N}
$$

Ni=número de observaciones por nivel

$N=$ muestra

$\mathrm{Pi}=$ elementos más favorables

Qi=elementos más desfavorables

$\mathrm{e}=$ error considerado

\section{CONCLUSIONES}

Esta investigación deja abierta la posibilidad de que el concepto de habitabilidad sea una variable de evaluación de las edificaciones sostenibles. Además, la habitabilidad orienta las diferentes necesidades de los usuarios hacia la sostenibilidad, dando la posibilidad de que la edificación se encamine a tener propiedades resilientes que le permitan responder a los diferentes agentes que afectan la habitabilidad hoy.

Por otro lado, la validación del modelo de simulación para el prototipo de software que se propuso permite abrir una puerta de investigación en el tema de la simulación discreta, área de trabajo que se ha trabajado muy poco en arquitectura y que ofrece un sinfín de oportunidades y de aplicaciones que hay que diseñar y perfeccionar.

El prototipo de software en este momento se convierte en un laboratorio de exploración para la generación de otro software que pueden conducir a una patente de utilidad y con ellos aplicarlo en el ejercicio profesional. Sería muy útil que los diseñadores y constructores de edificaciones puedan identificar las diferentes necesidades de los usuarios de una edificación a través de la modelación y la simulación de variables; como en el caso del cálculo de variables bioclimáticas y de flexibilidad en la vivienda, a partir de los cuales, se logró la evaluación de las diferentes necesidades de la edificación y además, los impactos en el ambiente.
Por otro lado, se concluye que la aproximación a partir del concepto de habitabilidad plantea la interrelación de variables desde las visiones sociocultural y ambiental, entendida esta última como un sistema. En el enfoque socioambiental se establece que los problemas de la habitabilidad en la vivienda $y$, en general, en cualquier tipo de edificación, son complejos.

Para terminar, en cuanto a la evaluación y validación del componente de simulación contenido en el prototipo de software se concluyó lo siguiente:

Un modelo de simulación pretende demostrar el comportamiento de la realidad, el proceso clásico consiste en simular procesos discretos a partir de la recolección de datos reales. El modelo puede quedar completo si se da este paso, debido a que el usuario final podría ayudar a descubrir las probabilidades reales, y no las teóricas en el estado del arte, el cual en la mayoría de los casos no describe la realidad.

La identificación de las probabilidades se debe hacer con la metodología propuesta luego de recolectar los datos en campo.

La simulación se podría utilizar para la gestión urbana, debido a que serviría para comprobar si la normatividad cumple el estándar y las necesidades de los usuarios, no sin antes hacer el muestreo, la recolección de datos y su análisis de parámetros.

Finalmente, se recomienda elaborar una evaluación y validación del modelo de habitabilidad orientado a la sostenibilidad (MHOS) y del método integral de diseño ambienta (MIDA), que permitan verificar la pertinencia en campo de las hipótesis planteadas y valorar su aplicabilidad.

\section{AGRADECIMIENTOS}

El equipo de investigación quiere agradecer el trabajo de los siguientes alumnos en el diseño del prototipo de software que ha permitido explorar, evaluar y validar los modelos propuestos por los diferentes investigadores: Camilo Pestana, Diego Andrés Apolinar Santos, Gerson Steevens Gaitán García, Daniel Alejandro Mora Salcedo, Giovanny Andrés Ninco Hernández, Cristian de Jesús Schotborgh López, Diego Hernando Torres Sarmiento y Sergio Vargas Poveda. 


\section{REFERENCIAS}

Alcaldía Mayor de Bogotá (2012). Ficha de Estadística Básica de Inversión Distrital EBI-D. Bogotá: Alcaldía Mayor de Bogotá.

Camacol (2009). Producción de vivienda de bajo costo. Conferencia presentada en Bogotá: Mesa VIS Diego Echeverry Campos / Universidad de los Andes.

Cameron, E. y Pettitt, A. N. (2012). Approximate Bayesian Computation for astronomical model analysis: a case study in galaxy demographics and morphological transformation at high redshift. Monthly Notices of the Royal Astronomical Society, 425 (1), 44-65. doi: 10.1111/j.1365-2966.2012.21371.x

Cortés, O. y Villar, M. (2013). Método Integral de Diseño Ambiental (MIDA). Revista Nodo, 8 (16), 15-21.

Cubillos-González, R. (2006). Vivienda social y flexibilidad en Bogotá. ¿Por qué los habitantes transforman el hábitat de los conjuntos residenciales? Revista Bitácora Urbano Territorial, 10, 124-135.

Cubillos-González, R. (2010). Sistema de gestión de información de proyectos de vivienda social (SGIPVIS). Revista de Arquitectura, 12, 88-99.

Cubillos-González, R. (2012a). Estudio y gestión de estándares mínimos de flexibilidad en la vivienda social en Bogotá. Revista de arquitectura, 14, 65-75.

Cubillos-González, R. (2012b). La tecnología sostenible aplicada al proyecto arquitectónico. Conferencia presentada en el Tercer Encuentro Internacional Ekotectura 2012 - Retos de la sostenibilidad en la ciudad de siglo XXI, Bogotá, Colombia. Academia Colombiana de Arquitectura y Diseño. Bogotá, Colombia.

Cubillos-González, R. y Rodríguez-Álvarez, C. (2013). Evaluación del factor de habitabilidad en las edificaciones sostenibles. Revista Nodo, 8 (15), 47-64.

Departamento Nacional de Planeación (DNP) (2009). Macroproyectos de interés social Estrategia ciudades amables - Plan nacional de desarrollo 2006-2010. Conferencia presentada en la Mesa VIS Diego Echeverry Campos / Universidad de los Andes. Bogotá: Departamento Nacional de Planeación.

Dotto, C. B. S., Kleidorfer, M., Deletic, A., Rauch, W., McCarthy, D. T. y Fletcher, T. D. (2011). Performance and sensitivity analysis of stormwater models using a Bayesian approach and long-term high resolution data. Environmental Modelling \& Software, 26 (10), 1225-1239. doi: http://dx.doi. org/10.1016/j.envsoft.2011.03.013.

Edwards, B. (2001). Guía básica de la sostenibilidad. Barcelona: Gustavo Gili.

Escallón, C. y Rodríguez, D. (2010a). Las preguntas por la calidad de la vivienda: ¿quién las hace?, ¿quién las responde? Dearq, 6, 6-19.
Escallón, C. y Villate, C. (2010b). Actualización del código de construcciones de Bogotá. Conferencia presentada en la Reunión del Concreto, septiembre 22 al 24 de 2010. Cartagena, Colombia.

Escallón, C. y Villate, C. (2010c). Convenio revisión código de construcción de Bogotá. Conferencia presentada en la Mesa VIS Diego Echeverry Campos / Universidad de los Andes, junio 6 de 2010. Bogotá, Colombia.

Fiksel, J., Eason, T. y Frederickson, H. (2012). A Framework for Sustainability Indicators at EPA. Washington D.C.: United States Environmental Protection Agency.

Janczura, J. y Weron, R. (2012). Efficient estimation of Markov regime-switching models: An application to electricity spot prices. AStA Advances in Statistical Analysis, 96 (3), 385407. doi: 10.1007/s10182-011-0181-2.

Kahrobaee, S. y Asgarpoor, S. (2013). A hybrid analytical-simulation approach for maintenance optimization of deteriorating equipment: Case study of wind turbines. Electric Power Systems Research, 104 (0), 80-86. doi: http://dx.doi.org/10.1016/j. epsr.2013.06.012.

Lai Chung, K. (1983). Elementary Probability Theory with Stochastic Processes. Madrid: Reverté.

Li, C., Singh, V. P. y Mishra, A. K. (2013). A bivariate mixed distribution with a heavy-tailed component and its application to singlesite daily rainfall simulation. Water Resources Research, 49 (2), 767-789. doi: 10.1002/ wrcr.20063

Liu, F., Li, X., Ding, Y., Zhao, H., Liu, X., Ma, Y. y Tang, B. (2013). A social network-based trust-aware propagation model for $\mathrm{P} 2 \mathrm{P}$ systems. Knowledge-Based Systems, 41 (0), 8-15. doi: http://dx.doi.org/10.1016/j. knosys.2012.12.005

Mateescu, R. y Serwe, W. (2013). Model checking and performance evaluation with CADP illustrated on shared-memory mutual exclusion protocols. Science of Computer Programming, 78 (7), 843-861. doi: http:// dx.doi.org/10.1016/j.scico.2012.01.003.

Ministerio de Ambiente, Vivienda y Desarrollo Territorial (MAVDT) (2009). El ABC del cambio climático en Colombia. Bogotá: Ministerio de Ambiente, Vivienda y Desarrollo Territorial / Instituto de Hidrología, Meteorología y Estudios Ambientales (IDEAM) / Koninkrijk der Nederlanden (Embajada de los Países Bajos en Colombia).

Naciones Unidas (1993). Agenda 21. Conference on Environment \& Development Rio de Janeiro, Brazil, 3 to 14 June 1992: United Nations.

Naciones Unidas (2012). Cómo desarrollar ciudades más resilientes - Un manual para líderes de los gobiernos locales. Ginebra: Naciones Unidas.
Pestana, C. (2013). Analizar y diseñar un sistema de gestión de información para proyectos de vivienda de interés social. Tesis de grado. Facultad de Ingeniería. Universidad Católica de Colombia.

Quintana, H. A. R. y Lizcano, F. R. (2007). Comportamiento resiliente de materiales granulares en pavimentos flexibles: estado del conocimiento. Ingenierías, 6 (11).

Ramírez, A. (2002). La construcción sostenible. Física y sociedad, 13, 30-33.

Rigaux Ancelet, C. S., Carlin, F., Nguyen-thé, C. y Albert, I. (2013). Inferring an Augmented Bayesian Network to Confront a Complex Quantitative Microbial Risk Assessment Model with Durability Studies: Application to Bacillus Cereus on a Courgette Purée Production Chain. Risk Analysis, 33(5), 877-892. doi: 10.1111/j.1539-6924.2012.01888.x

Sang, L., Zhang, C., Yang, J., Zhu, D. y Yun, W. (2011). Simulation of land use spatial pattern of towns and villages based on CA-Markov model. Mathematical and Computer Modelling, 54 (3-4), 938-943. doi: http://dx.doi. org/10.1016/j.mcm.2010.11.019

Serra, R. (2010). Arquitectura y climas. Barcelona: Gustavo Gili.

Sheaffer, R., Mendenhal, W. y Ott, L. (2007). Elementos del muestreo. Madrid: International Tomson Editores.

Solibakke, P. B. (2012). Scientific stochastic volatility models for the Salmon forward market: forecasting (un-)conditional moments. Aquaculture Economics \& Management, 16 (3), 222249. doi: 10.1080/13657305.2012.704618.

Trujillo, J. y Cubillos-, R. (2014). La simulación como herramienta del diseño y evaluación arquitectónica, paradigmas resueltos desde la ingeniería. Studiositas, 26.

Trujillo, J., González, E. y Velásquez, A. (2013). Hybrid model for making Tactical and Operational decisions in land transportation for the case of a perishable Supply Chain. Documento presentado en el 2nd International Symposium (1963-2013 - 50th Anniversary) - 24th National Conference on Operational Research; Hellenic Operational Research Society (HELORS), Atenas, Grecia.

Trujillo, J., Vallejo, J. y Becerra, M. (2010). Methodology Call-Centers's Simulation - Study case. Studiositas, 5 (3), 117-138.

Yang, H., Li, Y., Lu, L. y Qi, R. (2011). First order multivariate Markov chain model for generating annual weather data for Hong Kong. Energy and Buildings, 43 (9), 23712377. doi: http://dx.doi.org/10.1016/j. enbuild.2011.05.035 


\section{(A) CON RESPECTO A LOS AUTORES}

Para la Revista de Arquitectura, la postulación de un artículo indica que el o los autores certifican que conocen y aceptan la política editorial, para lo cual firmarán en original y remitirán el formato RevArq FP00 Carta de originalidad.

Para efectos de la autoría y coautoría de artículos se diferencian dos tipos "obra en colaboración" y "obra colectiva". La primera es aquella cuya autoría corresponde a todos los participantes al ser fruto de su trabajo conjunto. En este caso, se requiere el consentimiento de todos ellos para su divulgación. La obra colectiva es en la que, aunque participan diversos colaboradores, hay un autor que toma la iniciativa, la coordinación y realización de dicha obra. En estos casos, la autoría correspondería a dicha persona (salvo pacto en contrario) y sería suficiente únicamente con su autorización de divulgación.

En virtud de mantener el equilibro de las secciones y las mismas oportunidades para todos participantes, un mismo autor puede postular dos o mas artículos de manera simultanea, y previa evaluación de pares, la publicación se hará en volúmenes diferentes.

Se recomienda que el número de autores por artículo no sea superior a cinco integrantes y el orden en que se enuncien corresponda a los aportes de cada uno a la construcción del texto. Si se incluyen más personas se siguiere que sea en calidad de colaboradores o como parte de los agradecimientos. La Revista de Arquitectura respetará el orden en que figuren en el original remitido. La comunicación se establece con uno de los autores, quien a su vez será el responsable de informar a los demás colaboradores.

Una vez publicado el artículo, se envía al autor la versión impresa y digital, las cuales puede distribuir de manera libre respetando la licencia de acceso abierto y la integridad de la Revista de Arquitectura.

Para el caso del autoarchivo, si hay una versión previa (working paper - 'literatura gris' o pre-print) o una versión posterior (revisada o mejorada o post-print), el autor está en libertad de publicarlas en un sitio web o repositorios, siempre haciendo referencia a la publicación realizada en la Revista de Arquitectura.

\section{(A) ACCESO ABIERTO}

La Revista de Arquitectura, en su misión de divulgar la investigación y apoyar el conocimiento y discusión en las campos de interés, proporciona acceso libre, inmediato e irrestricto a su contenido de manera gratuita mediante la distribución de ejemplares impresos y digitales. Los interesados pueden leer, descargar, guardar, copiar y distribuir, imprimir, usar, buscar o referenciar el texto completo de los artículos o de la totalidad de la Revista de Arquitectura

Esta revista se acoge una licencia Creative Commons (CC) de Atribución - No comercial - Compartir igual, 4.0 Internacional: "E material creado puede ser distribuido, copiado y exhibido por terceros si se muestra en los créditos. No se puede obtener ningún beneficio comercial y las obras derivadas tienen que estar bajo los mismos términos de licencia que el trabajo original".

(i)(2) Para más información: http://

BY NO SA Co.creativecommons.org/tipos-de-licencias/

Las licencias CC se basan en el principio de la libertad creativa con fines académicos, científicos, culturales. Las licencias CC complementan el derecho de autor sin oponerse a este.

La Revista de Arquitectura es divulgada en centros y grupos de investigación, en bibliotecas y universidades y en las principales facultades de arquitectura, mediante suscripción anual o canje, este último se formaliza mediante el formato RevArq FP20 Canjes

Para aumentar su visibilidad e impacto de los artículos, se envían a bases de datos y sistemas de indexación y resumen (SIR) y asimismo pueden ser consultados y descargados en la página web de la revista.

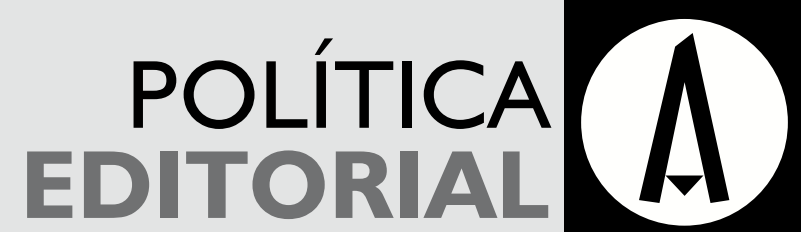

APRINCIPIOS ÉTICOS Y BUENAS PRÁCTICAS

La Revista de Arquitectura no tiene tarifa por procesamiento de artículos ni costos asociados al valor de página publicada.

Los artículos publicados en la Revista de Arquitectura son sometidos al cumplimiento de los principios éticos contenidos en las diferentes declaraciones y legislaciones sobre propiedad intelectual y derechos de autor específicos del país donde se realizó la investigación. En consecuencia, los autores de los artículos aceptados para publicar y que presentan resultados de investigaciones, deben firmar la declaración de originalidad, de cesión de derechos y de cumplimiento total de los principios éticos y las legislaciones específicas.

La Revista de Arquitectura se guía por las normas internacionales sobre propiedad intelectual y derechos de autor, y de manera particular el artículo 58 de la Constitución Política de Colombia, la Ley 23 de 1982 y el Acuerdo 172 del 30 de Septiembre de 2010 (Reglamento de propiedad intelectual de la UNIVERSIDAD CATÓLICA DE COLOMBIA).

Los autores, el editor, los miembros de los comités y los pares deben seguir las normas éticas internacionales (http://publicationethics.org) con el fin de evitar casos de fabricación, falsificación, omisión de datos y plagio.

La fabricación de resultados se ocasiona al mostrar datos inventados por los autores; la falsificación resulta cuando los datos son manipulados y cambiados a capricho de los autores; la omisión se origina cuando los autores ocultan deliberadamente un hecho o dato, y el plagio cuando un autor presenta como ideas propias, datos creados por otros. Los casos de plagio son los siguientes: copia directa de un texto sin entrecomillar o citar la fuente, modificación de algunas palabras del texto, paráfrasis y falta de agradecimientos. La revista se apoya en herramientas que detectan cualquiera de estos casos en los artículos postulados.

Una vez constatadas la Revista de Arquitectura podrá hacer públicas las malas prácticas científicas como plagio, falsificación o invención de datos, apropiación individual de autoría colectiva y publicación duplicada por parte del autor o los autores. El autor quedará impedido para postular artículos por dos años.

\section{A MANEJO DE LA INFORMACIÓN Y PRIVACIDAD}

HABEAS DATA

Para dar cumplimiento a lo previsto en el artículo 10 del Decreto 1377 de 2013, reglamentario de la Ley 1581 de 2012 y según el Acuerdo 002 del 4 de septiembre de 2013 de la UNIVERSIDAD CATÓLICA DE COLOMBIA, "por el cual se aprueba el manual de políticas de tratamiento de datos personales"

La UNIVERSIDAD CATÓLICA DE COLOMBIA, considerada como responsable y/o encargada del tratamiento de datos personales, manifiesta que los datos personales de los autores, integrantes de los comités y pares evaluadores, se encuentran incluidos en nuestras bases de datos; por lo anterior y en cumplimiento de las disposiciones legales vigentes, la Universidad solicitará siempre su autorización, para que en desarrollo de sus funciones propias como Institución de Educación Superior, en especial las relacionadas con la docencia, la extensión y la investigación, la UNIVERSIDAD CATÓLICA DE COLOMBIA pueda recolectar, recaudar, almacenar, usar, circular, suprimir, procesar, intercambiar, compilar, dar tratamiento, actualizar, transmitir y/o transferir a terceros países y disponer de los datos que le ha suministrado y que han sido incorporados en las bases de datos de todo tipo que reposan en la Universidad.

La UNIVERSIDAD CATÓLICA DE COLOMBIA queda autorizada, de manera expresa e inequívoca, en los términos señalados por el Decreto 1377 de 2013, para mantener y manejar la información de nuestros colaboradores (autores, integrantes de los diferentes comités y pares evaluadores), así mismo los colaboradores podrán ejercer sus derechos a conocer, actualizar, rectificar y suprimir sus datos personales, para lo cual se han dispuesto las siguientes cuentas de correo electrónico:

contacto@ucatolica.edu.co y revistadearquitectura@ucatolica.edu.co 
La Revista de Arquitectura. recibe de manera permanente artículos y los periodos de publicación son enero-diciembre de cada año. A medida que se van artículos recibiendo artículos se procesan.

El idioma principal es el español y como opcionales están definidos el inglés y el portugués; los textos pueden ser escritos y presentados en cualquiera de estos idiomas.

Los artículos postulados deben corresponder a las categorías universalmente aceptadas como producto de investigación, ser originales e inéditos y sus contenidos responder a criterios de precisión, claridad y brevedad.

Como punto de referencia se pueden tomar las tipologías y definiciones del Índice Bibliográfico Nacional, Publindex, para los artículos tipo 1,2 y 3 que se describen la continuación:

1) Artículo de investigación científica y tecnológica: documento que presenta, de manera detallada, los resultados originales de proyectos terminados de investigación. La estructura generalmente utilizada contiene cuatro apartes importantes: introducción, metodología, resultados y conclusiones.

\section{A INSTRUCCIONES PARA POSTULAR ARTÍCULOS}

Presentar el artículo mediante comunicación escrita dirigida al Editor de la Revista de Arquitectura (RevArq FP00 Carta de originalidad) ${ }^{1}$, en soporte digital debidamente firmada y una copia impresa (si es local o escaneada), adjuntando hoja de vida del autor (diligenciar el formato RevArq FP01 Hoja de Vida). En la comunicación escrita el autor debe expresar, que conoce y acepta la política editorial de la Revista de Arquitectura, que el artículo no está postulado para publicación simultáneamente en otra revistas u órganos editoriales y que -de ser aceptado- cede todos los derechos de reproducción y distribución del artículo a la UNIVERSIDAD CATÓLICA DE COLOMBIA Como editora de la revista.

Los artículos deben tener en cuenta las siguientes recomendaciones:

- En la primera página del documento se debe incluir

Título: en español e inglés y no exceder 15 palabras.

SUBтítulo: opcional, complementa el título o indica las principales subdivisiones del texto.

DATOS DEL AUTOR O AUTORES: nombres y apellidos completos, filiación institucional ( $\mathrm{Si}$ el artículo tiene patrocinio, financiación o apoyo de una institución o entidad). Como nota al pie (máximo 150 palabras): formación académica, experiencia profesional e investigativa, vinculación laboral, premios o reconocimientos, publicaciones representativas e información de contacto correo electrónico, dirección postal o numero telefónico.

DESCRIPCIÓN DEL PROYECTO DE INVESTIGACIÓN: en la introducción describir el tipo de artículo y brevemente el marco investigativo del cual es resultado y diligenciar el formato (RevArq FP02 Info Proyectos de Investigación)

RESUMEN:debe ser analítico, se redacta en un solo párrafo, da cuenta del tema, el objetivo, la metodología, los puntos centrales y las conclusiones, no debe exceder las 150 palabras y se presenta en españo e inglés (Abstract)

Palabras Clave: cinco palabras o grupo de palabras, ordenadas alfabéticamente y que no se encuentren en el título o subtítulo, deben presentarse en español e inglés (Key words), estas sirven para clasifica temáticamente al artículo. Se recomienda emplear principalmente palabras definidas en el tesauro de la Unesco http://databases.unesco.org thessp/ o en el tesauro de Arte \& Arquitectura (C) www.aatespanol.cl

- La segunda página y siguientes deben tener en cuenta estas recomendaciones:

El cuerpo del artículo generalmente se divide en: Introducción, Metodología, Desarrollo, Resultados y Discusión, y finalmente Conclusiones, luego se presentan las Referencias bibliográficas, Tablas, Leyendas de las Figuras y Anexos.

TexTO: Todas las páginas deben venir numeradas y con el título de artículo en la parte inferior (pie de página). Márgenes de $3 \mathrm{~cm}$ por todos los lados, interlineado doble, fuente, Arial o Times New Roman de 12 puntos, texto justificado. La extensión de los artículos debe estar alrededor de 5.000 palabras ( \pm 20 páginas, incluyendo gráficos, tablas, etc.); como mínimo 3.500 y máximo 9.000 palabras. Se debe seguir el estilo vigente y recomendado en el Manual para Publicación de la Asociación Americana de Psicología (APA). (Para mayor información http://www.apastyle.org).

CITAS Y NOTAS AL PIE: las notas aclaratorias o notas al pie no deben exceder cinco líneas o 40 palabras, de lo contrario estas deben ser incorporadas al texto general. Las citas pueden ser:

Corta (con menos de 40 palabras) se incorporan al texto y pueden ser: textuales (se encierran entre dobles comillas), parafraseo o resumen (se escriben en palabras del autor dentro del texto).

Cita textual extensa (mayor de 40 palabras) debe ser dispuesta en un renglón y un bloque independiente con sangrías y omitiendo las comillas, no olvidar en ningún caso la referencia del autor (Apellido, año, p. 00).

REFERENCIAS: como modelo para la construcción de referencias se emplea el siguiente:
2) Artículo de reflexión: documento que presenta resultados de investigación terminada desde una perspectiva analítica, interpretativa o crítica del autor, sobre un tema específico, recurriendo fuentes originales.

3) Artículo de revisión: documento resultado de una investigación terminada donde se analizan, sistematizan e integran los resultados de investigaciones publicadas o no publicadas, sobre un campo en ciencia o tecnología, con el fin de dar cuenta de los avances y las tendencias de desarrollo. Se caracteriza por presentar una cuidadosa revisión bibliográfica de por lo menos 50 referencias.

También se pueden presentar otro tipo de documentos diferentes a los anteriormente descritos como pueden ser: artículo corto, reporte de caso, revisión de tema, documento resultado de la revisión crítica de la literatura sobre un tema en particular, cartas al editor, traducción, documento de reflex ión no derivado de investigación, reseña bibliográfica así como proyectos de arquitectura o urbanismo, entre otros.
Libro

Autor-Apellidos-, A.A.-Nombres- (año de la publicación). Título de la obra. (Edición). Ciudad, País: Editorial.

Capítulo de un libro

Autor, A.A., \& Autor, B.B. (Año de la publicación). Título del capítulo. En A.A. Editor \& B.B. Editor (eds.), Título del libro (páginas del capítulo). Ciudad: Editorial.

\section{Publicación seriada (Revista)}

Autor, A.A., Autor, B.B., \& Autor, C.C. (Año de la publicación, incluya el mes y día de la publicación para publicaciones diarias, semanales o mensuales). Título del artículo. Título de la revista, diario, semanario, Volumen, (número), páginas.

Leyes, decretos, resoluciones, etc.

ey, decreto, resolución, etc, número (Año de la publicación, incluya el mes y día de la publicación). Título de la ley, decreto, resolución etc. Título de la publicación oficialmente. Ciudad, País

Artículo que se encuentra en una revista publicada en Internet Autor, A.A. \& Autor, B.B. (año, si se encuentra). Título del artículo. Título de la revista, volumen, (número). Recuperado de URL.

Siglas: en el caso de emplear siglas en el texto, cuadros, gráficos y/o fotografías, se deben proporcionar las equivalencias completas de cada una de ellas la primera vez que se empleen y encerrarlas entre corchetes [ ]. En el caso de citar personajes reconocidos se deben colocar nombres y/o apellidos completos, nunca emplear abreviaturas.

GRÁFICOS Y TABLAS: las figuras (gráficos, diagramas, ilustraciones, planos, mapas o fotografías) y las tablas deben contener número, título o leyenda explicativa relacionada con el tema del artículo que no exceda las 15 palabras (Figura 01 xxxxx, Tabla 01 xxxx, etc.) y la procedencia (autor $y / 0$ fuente, año, p. 00). Estos se deben incluir en el texto $y$ se deben citar de forma directa o entre paréntesis; se recomienda hacerlo mediante referencias cruzadas.

También se deben entregar en medio digital independiente del texto en formatos editables o abiertos. La numeración debe corresponder a la posición en el texto y según la extensión del artículo se deben incluir de 5 a 10 gráficos

El autor es el responsable de adquirir los derechos y/o las autorizaciones de reproducción a que haya lugar, para imágenes y/o gráficos tomados de otras fuentes, así como de entrevistas o material generado por colaboradores diferentes a los autores.

Fotografía: pueden ser entregadas en original para ser digitalizadas, de lo contrario se deben digitalizar con una resolución igual o superior a 300 dpi para imágenes a color y 600 para escala de grises. Los formatos de las imágenes pueden ser TIFF, PSD o JPG y deben cumplir con características expresadas en el punto anterior (gráficos)

Planimetría: se debe entregar la planimetría original en medio digital en lo posible en formato CAD y sus respectivos archivos de plumas o en PDF, de no ser posible se deben hacer impresiones en tamaño carta con las referencias de los espacios mediante numeración y lista adjunta. Deben tener escala gráfica, escala numérica, norte, coordenadas y localización. En lo posible no se deben textos, achurados o tramas.

Para más detalles, consultar el documento RevArq Parámetros para Autores Descripción en el portal web de la Revista de Arquitectura (www.ucatolica.edu.co).

\section{BENEFICIOS}

Como reconocimiento a los autores, se les hará envío postal de tres (3) ejemplares de la edición impresa sin ningún costo y entregada en la dirección consignada en el formato de hoja de vida (RevArq FP01) adicionalmente se les enviará el vínculo para la descarga de la versión digital. También se enviará una constancia informativa en la que se relaciona la publicación del artículo y de manera opcional se puede detallar las fechas del proceso editorial y el arbitraje realizado. 
La selección de pares evaluadores se realiza de acuerdo a los siguientes criterios:

- Afinidad temática

- Formación académica

- Experiencia investigativa y profesional

- Producción editorial en revistas similares y/o en libros resultado de investigación.

El proceso de arbitraje se basa en los principios de equidad e imparcialidad y en los criterios de calidad y pertinencia.

El desarrollo de la evaluación se realiza según el formato RevArq FP10 Evaluación de artículos calidad y las observaciones que el par considere necesarias en el cuerpo del artículo. En cualquiera de los conceptos que emita el par (aceptar, aceptar con modificaciones o rechazar) y como parte de la labor formativa y de comunidad académica, el par expondrá sugerencias para mejorar el documento. El par evaluador podrá solicitar una nueva relectura del artículo después de los ajustes realizados por el autor.

El par también deberá diligenciar el formato RevArq FP01 Hoja de Vida, con el fin de certificar y soportar el proceso de evaluación ante los SIR que así lo soliciten.

En el proceso de arbitraje se emplea el método doble ciego, los nombres de evaluador no serán conocidos por el autor y viceversa. Con el fin de garantizar el anonimato del autor, al artículo postulado se le han podido suprimir nombres, instituciones y/o imágenes que puedan ser asociadas de manera directa al autor.

Aunque se procura el anonimato, una vez recibida la invitación a evaluar el articulo, el par debe cerciorarse que no exista conflicto de intereses o alguna limitante que afecte la evaluación o que pueda ser vista como tal, (lazos familiares, amistad o enemistad, vínculos contractuales o laborales, posiciones éticas, etc), de presentarse esta situación se notificara al editor

Dada la confidencialidad del proceso de evaluación y considerando los derechos autor y de propiedad intelectual que pueda haber sobre el material que se entrega, el evaluador se compromete a mantener en absoluta reserva su labor, a limitar el uso de la obra entregada solo para el propósito de evaluación y a devolver la documentación que se le remite una vez realizada la evaluación.

El tiempo establecido para las evaluaciones es de máximo un (1) mes a partir de la confirmación de la recepción de la documentación. Ese plazo podrá ser modificado de mutuo acuerdo entre el editor y el par, siempre cuando no afecte la periodicidad de la revista, la impresión y/o el tiempo para emitir una respuesta al autor.

\section{BENEFICIOS}

Como retribución a los pares evaluadores, se les hará envío postal de un (1) ejemplar de la edición impresa sin ningún costo y entregada en la dirección consignada en el formato de hoja de vida. También si es de interés para el par, podrá hacer la solicitud de alguna de las publicaciones editadas y presentes en el catálogo de publicaciones de la UNIVERSIDAD CATÓLICA DE COLOMBIA, previa aprobación de la Editorial y sujeto a la disponibilidad.

Si lo desea tendrá derecho a solicitar una constancia de la colaboración en la evaluación de artículos, la cual solo contendrá el periodo en el cual se realizó la evaluación. También tendrá la posibilidad de aceptar o no la publicación de su nombre, nacionalidad y nivel máximo de formación en la página web de la Revista de Arquitectura en su calidad de colaborador.
El Comité Editorial de la Revista de Arquitectura es la instancia que decide la aceptación de los artículos postulados, el editor selecciona y clasifica solo los artículos que cumplan con los requisitos establecidos en las instrucciones para los autores.

Todos los artículos se someterán a un primer dictamen del Comité Editorial, el editor y de los editores de sección, teniendo en cuenta:

- Afinidad temática, relevancia del tema y correspondencia con las secciones definidas.

- Respaldo investigativo.

En caso de que los artículos requieran ajustes preliminares, este será devuelto al autor antes de ser remitidos a pares. En este caso el autor tendrá 15 días para remitir nuevamente el texto con los ajustes solicitados.

Después de la preselección se asignan mínimo dos pares evaluadores internos $y / 0$ externos especializados quienes emitirán su concepto utilizando el formato RevArq FP10 Evaluación de artículos calidad, se garantiza la confidencialidad y anonimato de autores y árbitros (modalidad doble ciego).

Del proceso de arbitraje se emite uno de los siguientes conceptos que son reportados al autor:

- (AA) Aceptar el artículo sin observaciones

- (AM) Aceptar el artículo con modificaciones: se podrá sugerir la forma más adecuada para una nueva presentación y se adjuntará la síntesis de los conceptos emitidos por los pares, el autor puede o no aceptar las observaciones según sus argumentos. Si las acepta, cuenta con quince (15) días para realizar los ajustes pertinentes.

- (RA) Rechazar el artículo: en este caso se entregará al autor un comunicado exponiendo las razones por las cuales se rechaza. En este caso, el autor puede volver a postular el artículo e iniciar nuevamente el proceso de arbitraje, siempre y cuando se evidencien los ajustes correspondientes.

En el caso de presentarse diferencias sustanciales y contradictorias en los conceptos de evaluación, el editor remitirá el artículo a un evaluador más o un miembro del Comité Editorial podrá asumir la tarea de actuar como el tercer árbitro, esto con el fin de tomar una decisión sobre la publicación del artículo.

El Comité Editorial se reserva el derecho de aceptar o no la publicación del material recibido. También se reserva el derecho de sugerir modificaciones de forma, ajustar las palabras clave o el resumen y de someterlo a corrección de estilo.

Cuando un artículo es aceptado para su publicación, los derechos de reproducción y divulgación son de la UNIVERSIDAD CATÓLICA DE COLOMBIA, lo cual se formaliza mediante la firma de la autorización de reproducción RevArq FP03 Autorización reproducción artículo. Esta autorización de uso no es exclusiva

\section{NOTAS ACLARATORIAS}

Aunque la recepción del material se notificará por correo electrónico en un plazo máximo de (8) ocho días, los procesos de evaluación, arbitraje, edición y publicación pueden tener un plazo máximo de (12) doce meses. A petición del autor, el editor informará sobre el estado del proceso editorial del artículo.

El editor de la Revista de Arquitectura es el encargado de establecer contacto entre los autores, árbitros, evaluadores y correctores, ya que estos procesos se realizan de manera anónima.

La Revista de Arquitectura publica un número limitado de artículos por volumen y busca el equilibrio entre las secciones, motivo por el cual aunque un artículo sea aceptado podrá quedar aplazado para ser publicado en una próxima edición, en este caso el autor estará en la posibilidad de retirar la postulación del artículo o de incluirlo en el banco de artículos del próximo volumen.

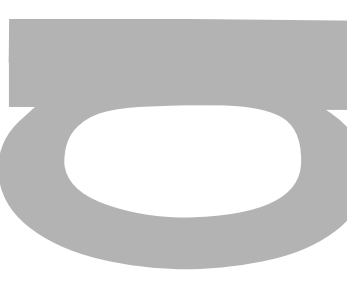

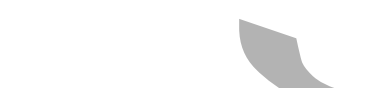
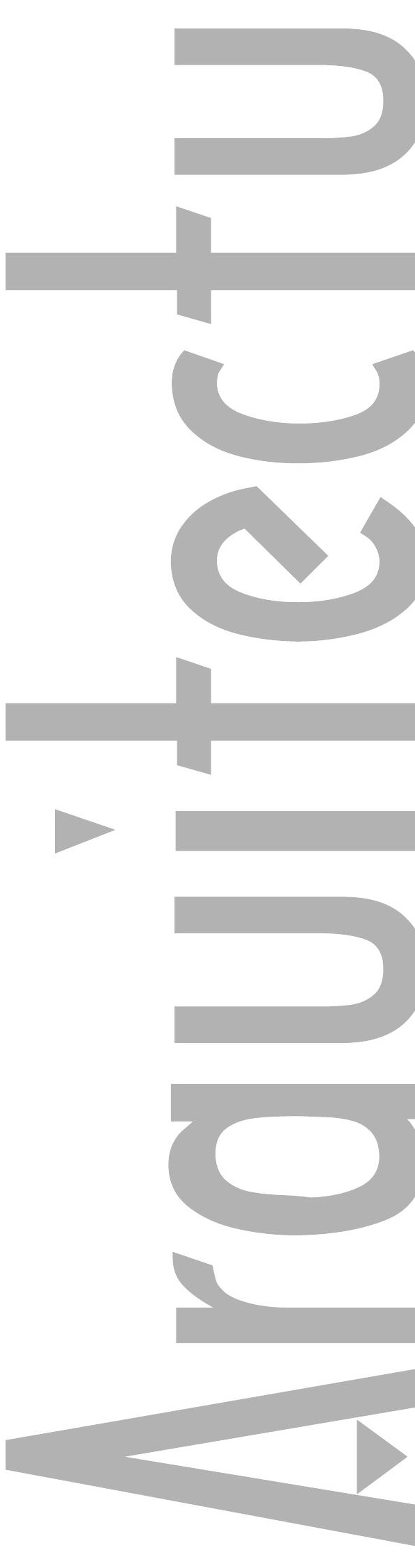
LOS USOS Y LA APROPIACIÓN DEL ESPACIO PÚBLICO PARA

EL FORTALECIMIENTO DE LA DEMOCRACIA

USES AND SPACE APPROPRIATION OF PUBLIC SPACE FOR THE

DEMOCRACY BUILD-UPABSTRACT

Pablo PÁramo, AndRea Milena Burbano

CIUDAD Y COMPROMISO CIUDADANO EN

LA HISTORIA DE OCCIDENTE

نे

CITY AND CITIZEN COMMITMENT IN WESTERN HISTORY

CARACTERIZACIÓN DEL MODELO DE APRENDIZAJE A

PARTIR DE LABORATORIOS DE DISEÑO CON ÉNFASIS EN

FACTORES SOCIALES

CHARACTERIZATION OF THE LEARNING MODEL BASED ON DESIGN

LABORATORIES WITH AN EMPHASIS ON SOCIAL FACTORS

\section{ÁLVARO JAVIER BOLAÑOS PALACIOS,}

ANÁLISIS URBANO Y FORMAL DEL EDIFICIO MIGUEL DE

AGUINAGA

URBAN AND FORMAL ANALYSIS OF MIGUEL DE AGUINAGA BUILDING

FELIPEVILLA MONTOYA, LEONARDO CORREA VELASQUEZ

VIVIENDAS DE EMERGENCIA EN URUGUAY

EMERGENCY HOUSING IN URUGUAY

JuAN José Fontana CABEZAS, PABlo Gustavo LaURino

CASTIGLIONI, MARIA VIRGINIA VILA RIVERO, LETICIA ANDREA BOTTI

AZAMBUYY

CUESTIONES DE MÉTODO CREATIVO

METAMORFOSIS Y CONCIENCIA MATERIAL EN LOS

PROCESOS CREATIVOS EN ARQUITECTURA

CREATIVE METHOD MATTERS

MetAMORPHOSIS AND MATERIAL CONSCIOUSNESS IN THE CREATIVE PROCESSES IN ARCHITECTURE

CARLOS IVÁN RUEDA PLATA

CRÍTICA SISTÉMICA

UN ENFOQUE HERMENÉUTICO DEL FENÓMENO ARQUITECTÓNICO

SYSTEMIC CRITICISM - AN HERMENEUTICAL STANDPOINT OF THE

ARCHITECTURAL PHENOMENON

ESKA ELENA SOLANO MENESES

TRADUCCIÓN DEL DISEÑO CONCURRENTE AL PROYECTO DE ARQUITECTURA

TRANSLATION OF THE CONCURRENT DESIGN TO THE

ARCHITECTURE PROJECT

Luis Álvaro Flórez Millán, Jairo Hernan OVALLE Garay,

LEONEL AUGUSTO FORERO LA ROTTA

EFICIENCIA DE ESTRATEGIAS DE ENFRIAMIENTO

PASIVO EN CLIMA CÁLIDO SECO

EFFICIENCY OF PASSIVE COOLING STRATEGIES IN HOT DRY WEATHER

LUIS CARLos HerRERA SOSA

SIMULACIONES AMBIENTALES PARA LA SELECCIÓN DE MATERIALES EN DISEÑO DE ALOJAMIENTOS TEMPORALES EN CLIMAS TROPICALES

ENVIRONMENTAL SIMULATIONS FOR MATERIAL SELECTION

IN TEMPORARY HOUSING DESIGN IN TROPICAL WEATHER

CONDITIONS

$$
\text { SARA LUCIANI M. }
$$

A INFLUÊNCIA DAS PRATELEIRAS DE LUZ NO

APROVEITAMENTO DA LUZ NATURAL SOB OBSTRUÇÃO EXTERNA

THE INFLUENCE OF LIGHT SHELVES IN THE HIGH-PERFORMANCE

USE OF NATURAL LIGHT UNDER EXTERNAL OBSTRUCTION

RICARDO NACARI MAIOLI, MARIANI DAN TAUFNER,

CRISTINA ENGEL DEALVAREZ

LA HABITABILIDAD COMO VARIABLE DE DISEÑO DE

$\pm \quad$ EDIFICACIONES ORIENTADAS A LA SOSTENIBILIDAD

$=$ Habitability AS DESIGN VARIABle OF BuILDINGS HEADING

Uं TOWARDS SUSTAINABILITY

¿ ROLANDO ARTURO CUBILLOS GONZÁLEZ, JOHANNA TRUJILLC,

Oscar AlFonso Cortés CELy, Claudia Milena Rodrígueż

ÁlVAREZ, MAYERLY ROSA VILLAR LOZANO

LA SOSTENIBILIDAD DE LA VIVIENDA TRADICIONAL:

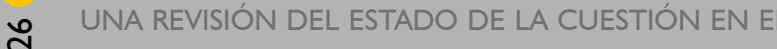
ㄴ MUNDO

U TRADITIONAL Housing SUSTAINABILITY: A REVIEW OF THE STAT ¿ OF THE ART IN THE WORLD

RIGOBERTO LÁRRAGA LARA, MIGUEL AGUILAR ROBLEDO,

\section{HIFHA \\ ( $5^{\circ}$ CONCURSO DE DISEÑO EN ACERO PARA ESTUDIAN-}

m TES DE ARQUITECTURA EN COLOMBIA - 2014

ن் SEGUNDO PUESTO. MEMORIA

a 5TH STEEL DESIGN ARCHITECTURE STUDENT COMPETITION IN COLOMBIA -20I4

\section{DIEGO ALEJANDRO MORA CASAS, JUAN CAMILO RI}
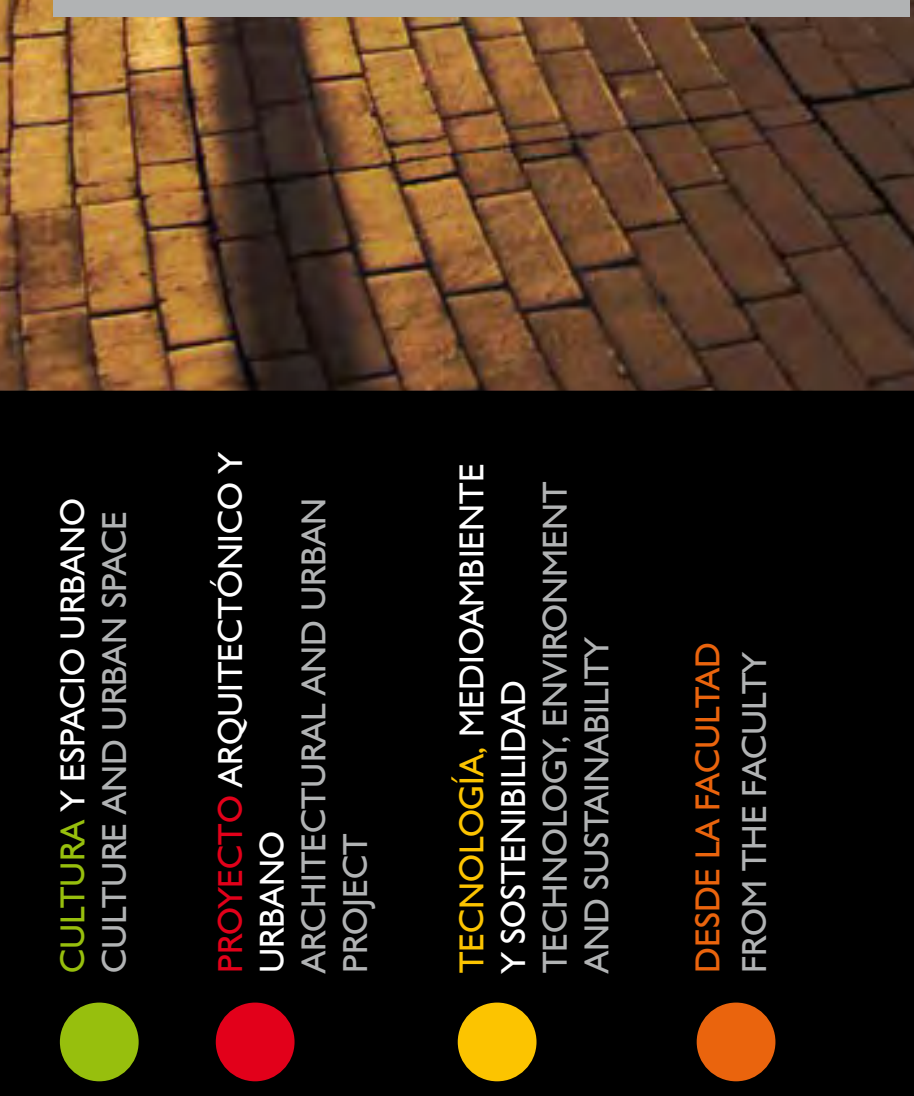

La Revista de Arquitectura es arbitrada e indexada y está presente en:

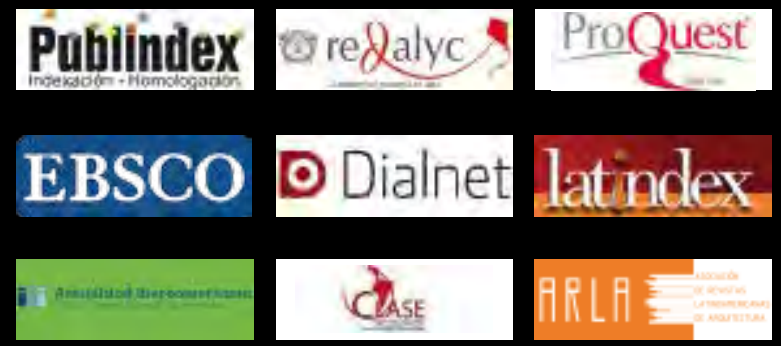

f

REVISTA DE ARQUITECTURA - UNIVERSIDAD CATOLICA DE COLOMBIA
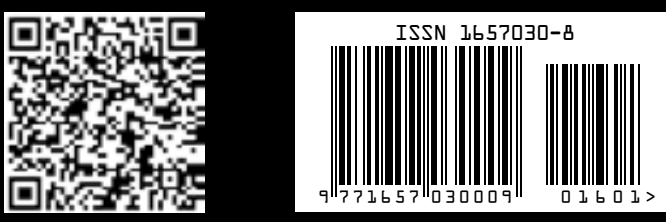